\title{
The Computation of Characteristic Classes of Lattice Gauge Fields
}

\author{
Anthony V. Phillips ${ }^{1 \star}$ and David A. Stone ${ }^{2 \star \star}$ \\ ${ }^{1}$ State University of New York at Stony Brook, Stony Brook, NY 11784, USA \\ 2 Brooklyn College, City University of New York, Brooklyn, NY 11210, USA
}

\begin{abstract}
A $G L(p, \mathbf{C})$-valued lattice gauge field $\mathbf{u}$ on a simplicial complex determines a principal $G L(p, \mathbf{C})$-bundle $\xi$ if the plaquette products are sufficiently small with respect to the maximum distortion coefficient of the transporters. A representative cocycle $c_{q}$ for the $q^{\text {th }}$ Chern class of $\xi$ can be computed on each $2 q$-simplex $\sigma$ by taking $c_{q}(\sigma)$ to be the intersection number of a certain singular $2 q$-cube $M_{\sigma}$ with a Schubert-type variety $\Sigma_{q}$ in the space of all $p \times p$ matrices. This reduces to the solution of polynomial equations with coefficients coming from $\mathbf{u}$ and thus avoids numerical integration or cooling-type procedures. An application of this method is suggested for the computation of the topological charge of an $S U(3)$-valued lattice gauge field on a 4-complex.
\end{abstract}

\section{Introduction}

This work grew out of our earlier research in the topology of lattice gauge fields $[23,24]$, which in turn was inspired by the work of Martin Lüscher [17]. There we gave algorithms for the computation of the characteristic numbers of $U(1)$ and $S U(2)$-valued lattice gauge fields on triangulated 2 and 4-dimensional manifolds. Here we examine the problem of computing characteristic classes of lattice gauge fields with values in $G L(p, \mathbf{C})$, for arbitrary $p$, defined on simplicial complexes of arbitrary dimension. In particular this work could be used as the basis of a new algorithm for the evaluation of the topological charge of an $S U(3)$-valued lattice gauge field on a 4-dimensional complex, a problem that has recently been examined from a wide variety of angles $[3,7,8,10,13,14,15,22]$, some of them reviewed in [12] along with the physical context of the problem. In our $S U(2)$ work we were able to exploit the extremely simple geometry of the group: since geodesics on a 3 -sphere are its intersections with 2-planes in 4-space, most questions about relative

* Partially supported by NSF grant DMS 8607168

$\star \star$ Partially supported by PSC-CUNY and by NSF grant DMS 8805485 
position can be immediately settled by linear algebra. Higher $S U(N)$ have a more complicated geometry; this led us to consider them as embedded in $G L(N, \mathbf{C})$, where the local geometry is linear. Also, for $S U(2)$-bundles on a 4-complex, the only characteristic class is the second Chern class $c_{2}$; this is the obstruction to a section in a bundle with fibre the 3 -sphere and can be calculated directly from this property. In general an $S U(N)$-bundle on a $d$-dimensional complex can have characteristic classes $c_{2}, c_{4}$, etc. obstructing sections in bundles with more complicated fibres (see [21, Problem 14-C]; this led us to devise a different, non-obstruction-theoretic method which applies equally well to any of them.

We take as data a triangulation $\Lambda$ of a topological space $X$, a local ordering $\mathbf{o}$ of the vertices of $\Lambda$, and a $G L(p, \mathbf{C})$-valued lattice gauge field $\mathbf{u}$ on $\Lambda$. Our earlier work was based on the extension of $\mathbf{u}$, by interpolation, to a principal coordinate bundle [26]. Here instead we define from u a $G L(p, \mathbf{C})$-valued parallel transport function $\mathbf{V}$ on $\Lambda$, a concept which is closely related to a twisted cochain [4]. This construction is possible if $\mathbf{u}$ is "continuous," i.e. if the plaquette products of $\mathbf{u}$ are sufficiently close to the identity.

Our main contribution is the explanation of how a continuous lattice gauge field $\mathbf{u}$ extends to a parallel transport function $\mathbf{V}$; how a parallel transport function $\mathbf{V}$ can be used to define a bundle $\xi$ (we show that if $\mathbf{u}$ is "strictly continuous," then $\xi$ does not depend on how $\mathbf{u}$ was extended to $\mathbf{V}$ ); and how generically $\mathbf{V}$ can be used directly to yield integer-valued cocycles on $\Lambda$ representing the Chern classes of $\xi$.

Here is an outline of the rest of this article. To each parallel transport function $\mathbf{V}$ we associate (in Sect. 2) a canonical pseudosection $\mathbf{H}$, which gives a specific classifying map

$$
f: X \rightarrow B_{\Delta} G,
$$

where $G$ is short for $G L(p, \mathbf{C})$, and $B_{\Delta} G$ is the base of Milnor's model of the universal $G$-bundle [19]. This map defines the principal $G$-bundle $\xi$ determined by $\mathbf{V}$. In Sect. 3 we show how $\mathbf{V}$ can be constructed from a lattice gauge field $\mathbf{u}$, and examine the dependence on $\mathbf{u}$ of the bundle $\xi$ so determined. Our computation scheme for characteristic classes is presented in Sect. 4. There we go from $f$ to a map taking $X$ to the standard (Grassmannian) classifying space, where the integral Chern classes are represented by canonical dual varieties, the Schubert cycles. We show how to compute intersections with Schubert cycles locally, and, when $\mathbf{V}$ is defined from $\mathbf{u}$ as above, in terms only of $\mathbf{u}$. It will turn out that computing these intersection numbers amounts to solving polynomial equations of a simple type, which we describe explicitly in Sect. 5 for the cases $U(1), S U(2)$ and $S U(3)$. Proofs of some of the more technical propositions are given in Sect. 6 , and the equivalence between parallel transport functions and principal coordinate bundles is proved in the Appendix.

The calculation from $\mathbf{u}$ of cochains representing characteristic classes can be applied (exactly as explained in the Introduction of [24]) to give a new algorithm, an (integral) alternate to the Chern-Weil construction, for the local computation of the characteristic classes of $G L(p, \mathbf{C})$-bundles with connections. 


\section{Combinatorial Preliminaries}

1.1 Let $C^{r}$ be the standard $r$-cube in $\mathbf{R}^{r}$, that is,

$$
C^{r}=\left\{\left(s_{1}, \ldots, s_{r}\right) \mid 0 \leqq s_{i} \leqq 1\right\} .
$$

For $j=1, \ldots, r$ the faces $\partial^{j} C^{r}$ and $\partial_{j} C^{r}$ are defined by the equations $s_{j}=1$ and $S_{j}=0$ respectively.

1.2 Let $\Delta^{r}$ be the standard $r$-simplex in $\mathbf{R}^{r}$, that is,

$$
\Delta^{r}=\left\{t_{0} \mathbf{e}_{0}+\cdots+t_{r} \mathbf{e}_{r}\right\}
$$

where $\mathbf{e}_{0}$ is the origin $(0, \ldots, 0), \mathbf{e}_{i}$ is the $i^{\text {th }}$ standard basis vector and $t_{0}+\cdots+t_{r}=1$. We call $\left(t_{0}, \ldots, t_{r}\right)$ the barycentric coordinates of $t_{0} \mathbf{e}_{0}+\cdots+t_{r} \mathbf{e}_{r}$. More generally, we use letters $i, j, \ldots$ to denote vertices of a simplicial complex $\Lambda$, and the notation $\sigma=\left\langle i_{0}, \ldots, i_{r}\right\rangle$ to indicate that $\sigma$ is a simplex of $\Lambda$, with those vertices. In contexts where $\sigma$ is being considered in isolation from $\Lambda$, we often write simply $\sigma=\langle 0, \ldots, r\rangle$.

Let $\partial_{j} \Delta^{r}=\left\langle\mathbf{e}_{0}, \ldots, \hat{\mathbf{e}}_{j}, \ldots, \mathbf{e}_{r}\right\rangle, \quad j=0, \ldots, r$.

Definition 1.3. We define the standard projection $\pi^{C^{r}}: C^{r} \rightarrow \Delta^{r}$ by induction on $r$; see Fig. 1.
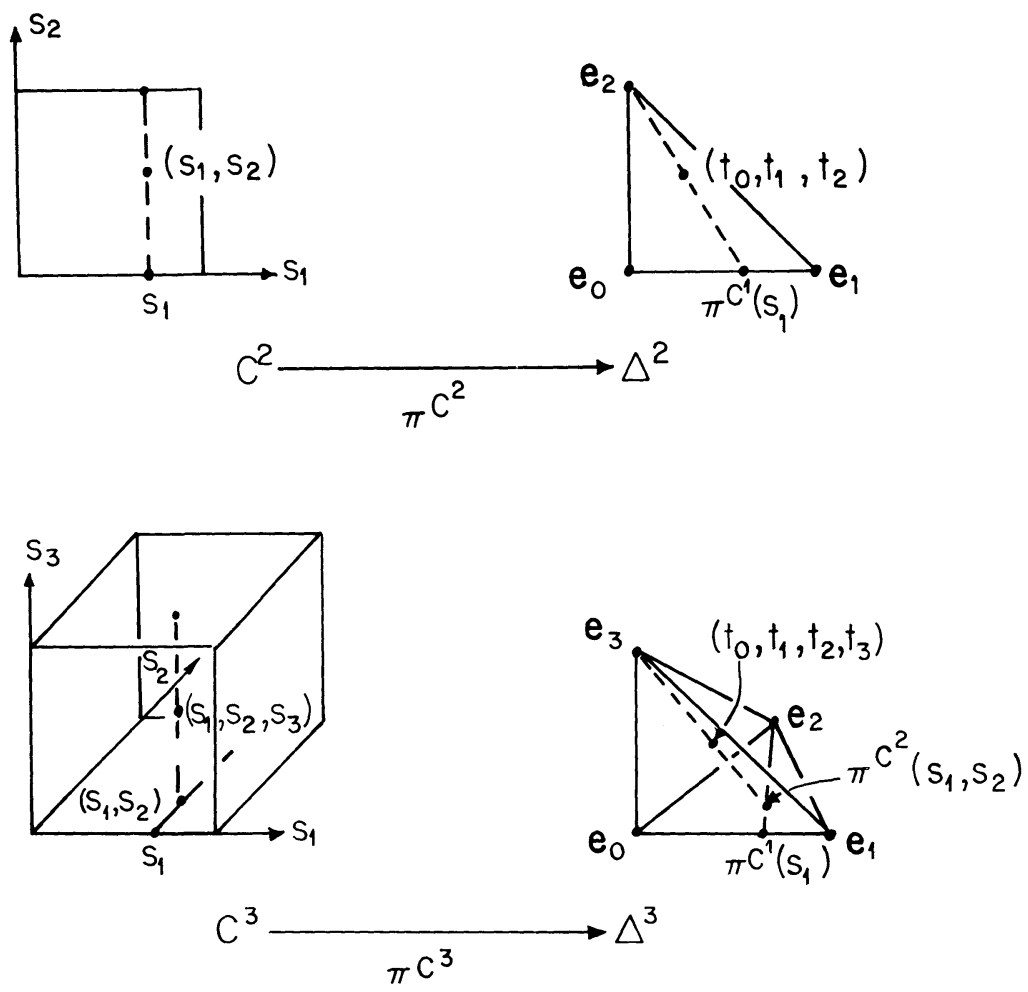

Fig. 1. The standard projection $\pi^{c^{r}}: C^{r} \rightarrow \Delta^{r}$ 
For $r=0$, the definition is trivial. Now assume that $\pi^{C^{r-1}}: C^{r-1} \rightarrow\left\langle\mathbf{e}_{0}, \ldots, \mathbf{e}_{r-1}\right\rangle$ has been defined. We identify $C^{r-1}$ with $\partial_{r} C^{r}$ and set

$$
\pi^{C^{r}}\left(s_{1}, \ldots, s_{r}\right)=\left(1-s_{r}\right) \pi^{c^{r-1}}\left(s_{1}, \ldots, s_{r-1}\right)+s_{r} \cdot \mathbf{e}_{r} .
$$

In terms of coordinates, $\pi^{C^{r}}\left(s_{1}, \ldots, s_{r}\right)=\left(t_{0}, \ldots, t_{r}\right)$, where

$$
\begin{array}{cr}
t_{0}=\left(1-s_{1}\right)\left(1-s_{2}\right) \cdots\left(1-s_{r}\right) \\
t_{1}= & s_{1}\left(1-s_{2}\right) \cdots\left(1-s_{r}\right) \\
t_{2}= & s_{2} \cdots\left(1-s_{r}\right) \\
\vdots & \\
t_{r}= &
\end{array}
$$

1.4 Orderings. A simplicial complex $\Lambda$ is locally ordered by giving a partial ordering $\mathbf{0}$ of its vertices in which the vertices of each simplex are totally ordered. We shall usually refer to "the locally ordered simplicial complex $\Lambda$ " and mention o only when necessary. For example, the first barycentric subdivision of any simplicial complex has a natural local ordering: if $\hat{\tau}$ and $\hat{\sigma}$ are barycenters of $\tau$ and $\sigma$ respectively, then $\hat{\tau} \prec \hat{\sigma}$ if $\tau$ is a face of $\sigma$. A local ordering $\mathbf{o}$ of a finite set can be refined to a total ordering: start with any o-minimal vertex; and inductively select any vertex which is o-minimal among those not yet selected.

An ordering of the vertices of an $r$-simplex $\sigma$ determines a unique orderrespecting linear homeomorphism $\sigma \rightarrow \Delta^{r}$ and picks out faces $\partial_{j} \sigma$ corresponding to the similarly labelled faces of $\Delta^{r}$.

1.5 Notation. Consider a simplex $\sigma$ in $\Lambda$, say $\sigma=\left\langle i_{0}, \ldots, i_{r}\right\rangle$, with vertices so ordered. To $\sigma$ we associate two cubes in $\mathbf{R}^{\infty}$, labelled $C_{\sigma}=C_{\sigma}^{r}$ and $c_{\sigma}=c_{\sigma}^{r-1}$, as follows:

$$
\begin{gathered}
C_{\sigma}^{r}=\left\{0 \leqq s_{i_{1}} \leqq 1, \ldots, 0 \leqq s_{i_{r}} \leqq 1\right\}, \\
c_{\sigma}^{r-1}=\left\{0 \leqq s_{i_{1}} \leqq 1, \ldots, 0 \leqq s_{i_{r-1}} \leqq 1\right\},
\end{gathered}
$$

and all the other coordinates are 0 .

In particular, if $\operatorname{dim} \sigma=0$, set $C_{\sigma}^{0}=\overrightarrow{0} \in \mathbf{R}^{\infty}$ and $c_{\sigma}^{-1}=\varnothing$.

1.6. The order-preserving homeomorphism mentioned above, together with a similar one from $C^{r}$ to $C_{\sigma}^{r}$, combine with the standard projection of Definition 1.3 to define a standard projection $\pi^{C_{\sigma}^{r}}: C_{\sigma}^{r} \rightarrow \sigma$.

1.7 Milnor's Universal G-Bundle. For a Lie group $G$, let $\gamma_{\Delta} G=\left(\pi_{\Delta}: E_{\Delta} G \rightarrow B_{\Delta} G\right)$ be Milnor's model of a principal $G$-bundle [19]. Here $E_{\Delta} G=G_{0} * G_{1} * \cdots$, the join of countably many copies of $G$. A point of $E_{\Delta} G$ is represented as $\sum_{i=0}^{\infty} t_{i} g_{i}$, where $g_{i} \in G_{i}, 0 \leqq t_{i} \leqq 1$, all but finitely many $t_{i}$ are 0 , and $\sum t_{i}=1$. $G$ acts on the left on $E_{\Delta} G$ by $g^{\prime} \cdot\left(\sum t_{i} g_{i}\right)=\sum t_{i}\left(g^{\prime} \cdot g_{i}\right)$, and $B_{\Delta} G$ is the quotient space. Note for future reference that the map $\pi^{\infty}: E_{\Delta} G \rightarrow \Delta^{\infty}=\mathbf{e}_{0} * \mathbf{e}_{1} * \cdots$ defined by $\pi^{\infty}\left(\sum t_{i} g_{i}\right)=\sum t_{i} \mathbf{e}_{i}$ is constant on $G$-equivalence classes and so defines a projection $p: B_{\Delta} G \rightarrow \Delta^{\infty}$. It is convenient to use the vectors $\mathbf{e}_{i}$ to index the various copies of $G$, by identifying $\mathbf{e}_{i}$ 
with the identity element in the $i^{\text {th }}$ copy; thus we will write $\sum t_{i} g_{i}$ as $\sum t_{i} g_{i} \mathbf{e}_{i}$, where now all $g_{i} \in G$.

\section{Parallel Transport Functions and their Canonical Pseudosections, Principal Bundles, and Classifying Maps}

We start with a finite simplicial complex $\Lambda$ and a partial ordering $\mathbf{o}$ of its vertices. For notational convenience we will embed $\mathbf{o}$ in a total ordering, and renumber the vertices $0,1,2, \ldots$ accordingly. (We will be using the consequent identification of $\Lambda$ with a subset of $\Delta^{\infty}$.)

Definition 2.1. A parallel transport function over a locally ordered simplicial complex $\Lambda$ consists of a family $\mathbf{V}$ of maps of cubes into $G$, one $V_{\sigma}: c_{\sigma}^{r-1} \rightarrow G$ for each $r$-simplex $\sigma$ of $\Lambda, r \geqq 1$, such that, for every $\sigma=\left\langle i_{0}, \ldots, i_{r}\right\rangle$ the following compatibility conditions hold:

$$
\begin{aligned}
& V_{\sigma}\left(s_{i_{1}}, \ldots, s_{i_{p-1}}, s_{i_{p}}=1, s_{i_{p+1}}, \ldots, s_{i_{r-1}}\right) \\
& \quad=V_{\left\langle i_{0}, \ldots, i_{p}\right\rangle}\left(s_{i_{1}}, \ldots, s_{i_{p-1}}\right) \cdot V_{\left\langle i_{p}, \ldots, i_{r}\right\rangle}\left(s_{i_{p+1}}, \ldots, s_{i_{r-1}}\right), \\
& \quad V_{\sigma}\left(s_{i_{1}}, \ldots, s_{i_{p-1}}, s_{i_{p}}=0, s_{i_{p+1}}, \ldots, s_{i_{r-1}}\right) \\
& \quad=V_{\left\langle i_{0}, \ldots, i_{p-1}, i_{p+1}, \ldots, i_{r-1}\right\rangle}\left(s_{i_{1}}, \ldots, s_{i_{p-1}}, s_{i_{p+1}}, \ldots, s_{i_{r-1}}\right) .
\end{aligned}
$$

For $\sigma=\left\langle i_{0} i_{1}\right\rangle$ a 1-simplex, we will abbreviate $\left.V_{\left\langle i_{0} i_{1}\right\rangle}\right\rangle(\overrightarrow{0})$ to $v_{\left\langle i_{0} i_{1}\right\rangle}$. In particular the compatibility conditions for a 2-simplex become

$$
\begin{gathered}
V_{\left\langle i_{0} i_{1} i_{2}\right\rangle}(1)=v_{\left\langle i_{0} i_{1}\right\rangle} \cdot v_{\left\langle i_{1} i_{2}\right\rangle}, \\
V_{\left\langle i_{0} i_{1} i_{2}\right\rangle}(0)=v_{\left\langle i_{0} i_{2}\right\rangle} .
\end{gathered}
$$

2.2 Remark. The notion of parallel transport function (p.t.f.) is a useful starting point for many basic structures in the topology and geometry of principal bundles. a) A parallel transport function includes a lattice gauge field (see Definition 3.2), namely that one defined by $u_{i_{0} i_{1}}=v_{\left\langle i_{0} i_{1}\right\rangle}$ and $u_{i_{1} i_{0}}=\left(v_{\left\langle i_{0} i_{1}\right\rangle}\right)^{-1}$ for $i_{0}<i_{1}$ in the ordering.

b) Conversely, a lattice gauge field $\mathbf{u}$ which is continuous (i.e. satisfies a certain bound, on its plaquette products; see Definition 3.7) determines a p.t.f.

c) A p.t.f. can be understood as a set of transition functions for a principal bundle, as follows. Reinterpret $c_{\sigma}^{r-1}$ as $\sigma \cap C_{0} \cap C_{r}$, where $C_{0}$ and $C_{r}$ are the dual cells to the lowest- and highest-ordered vertices of $\sigma$, respectively. Then the coordinates in $c_{\sigma}^{r-1}$ match the "modified barycentric coordinates" [24] in $\sigma$, and the $V$ 's can be interpreted as giving a set of $G$-valued transition functions on the interfaces of the dual cells of the vertices. The first compatibility condition is the cocycle condition, and the second guarantees coherence when passing from a $\sigma$ to a neighboring $\sigma^{\prime}$. Details are given in the Appendix.

d) The concept of a p.t.f. is closely related to one of the standard models used in algebraic topology to represent a fibre bundle. Let $\Lambda_{*}$ denote the chain complex generated by $\Lambda$, and $\mathscr{G}_{*}$ the singular chain complex of $G$. According to Brown [4] for any principal $G$-bundle $\xi=(\pi: E \rightarrow X)$ over $X=|\Lambda|$ one can equip $\mathscr{G}_{*} \otimes \Lambda$ with 
a new differential $\partial_{\phi}$ to obtain a chain-complex model of $E$. Brown derives $\partial_{\phi}$ directly from what he calls a twisting cochain $\phi_{*}$ for $\xi$. It turns out that a twisting cochain is essentially the same thing as a p.t.f., so that a p.t.f. $\mathbf{V}$ on $\Lambda$ leads almost immediately to the chain-complex model $\left(\mathscr{G}_{*} \otimes \Lambda, \partial_{\phi}\right)$ for the total space of a $G$-bundle. (This is the same bundle that we will associate to $\mathbf{V}$ by a construction more appropriate for our calculations.) More specifically, consider the standard subdivision of the $r$-cube $C^{r}$ into $r$ ! oriented $r$-simplexes $\tau_{\alpha}$, each with the same orientation as $C^{r}$. These simplexes are indexed by permutations $\alpha$ of $\{1, \ldots, r\}$; the vertices of $\tau_{\alpha}$ are $\mathbf{e}_{0}, \mathbf{e}_{\alpha(1)}, \mathbf{e}_{\alpha(1)}+\mathbf{e}_{\alpha(2)}$, etc. For $\operatorname{dim} \sigma=r \geqq 1$, the map $V_{\sigma}$ defines a chain $\psi_{\sigma}^{r-1} \in \mathscr{G}_{r-1}$ by $\psi_{\sigma}^{r-1}=\left.\sum_{\alpha} V_{\sigma}\right|_{\tau_{\alpha}}$. Then $\phi_{*}$ is the sequence of chain maps $\phi_{r}: \Lambda_{r} \rightarrow \mathscr{G}_{r-1}$ given by $\phi_{1}(\sigma) \stackrel{\alpha}{=} \psi_{\sigma}^{0}-e$ (here $e$, strictly speaking, represents the 0 -chain with image the identity element of $G)$ and $\phi_{r}(\sigma)=\psi_{\sigma}^{r-1}$, for $r>1$.

e) If $G$ is a Lie group, $X=|\Lambda|$ a smooth manifold, $\xi=(\pi: E \rightarrow X)$ a principal $G$-bundle, and $\omega$ a connection in $\xi$, then, by using parallel transport with respect to $\omega$ along certain short paths, we obtain a p.t.f. as follows. (Thus the p.t.f. emulates the role played by a connection, but on the small scale rather than the infinitesimal.)

For each vertex $\langle i\rangle$ choose an identification of the fiber over $\langle i\rangle$ with $G$; let $\mathbf{e}_{i}$ be the point in that fiber corresponding to the identity in $G$. Let $\mathbf{o}$ be a local ordering of the vertices of $\Lambda$. Let $\sigma=\left\langle i_{0}, \ldots, i_{r}\right\rangle \in \Lambda$, with vertices so ordered $(r \geqq 1)$, and in $\sigma$ pick points $P_{1}, \ldots, P_{r-1}$ so that

- $P_{1}$ is on the line segment from $\left\langle i_{0}\right\rangle$ to $\left\langle i_{1}\right\rangle$ (in the affine structure on $\sigma$ ): $P_{1}=\left(1-s_{1}\right)\left\langle i_{0}\right\rangle+s_{1}\left\langle i_{1}\right\rangle$;

- $P_{2}$ is on the line segment from $P_{1}$ to $\left\langle i_{2}\right\rangle: P_{2}=\left(1-s_{2}\right) P_{1}+s_{2}\left\langle i_{2}\right\rangle$; and so on until

- $P_{r-1}=\left(1-s_{r-1}\right) P_{r-2}+s_{r-1}\left\langle i_{r-1}\right\rangle$.

Then let $\alpha$ be the piecewise linear path from $\left\langle i_{0}\right\rangle$ through $P_{1}, P_{2}, P_{r-1}$, to $\left\langle i_{r}\right\rangle$. This path is determined uniquely (up to parametrization) by the $r-1$ numbers $s_{1}, s_{2}, \ldots, s_{r-1}$. We may now define $V_{\sigma}: c_{\sigma}^{r-1} \rightarrow G$ by the rule: $V_{\sigma}\left(s_{1}, \ldots, s_{r-1}\right) \cdot \mathbf{e}_{i_{r}}=$ the result of parallel transport of $\mathbf{e}_{i_{0}}$ along the corresponding $\alpha$. The collection of $V_{\sigma}$ 's so defined, for $\sigma \in \Lambda, \operatorname{dim} \sigma \geqq 1$, forms a parallel transport function over $\Lambda$. To check the compatibility condition

$$
\left.V_{\sigma}\right|_{s_{p}=1}=V_{\left\langle i_{0}, \ldots, i_{p}\right\rangle} \cdot V_{\left\langle i_{p}, \ldots, i_{r}\right\rangle},
$$

it is sufficient to remark that the corresponding paths all have $P_{p}=\left\langle i_{p}\right\rangle$; each can be written uniquely as the composition $\alpha^{\prime} \circ \alpha^{\prime \prime}$ of two paths, where $\alpha^{\prime}$, corresponding to parameters $s_{1}, \ldots, s_{p-1}$, runs from $\left\langle i_{0}\right\rangle$ to $\left\langle i_{p}\right\rangle$ in $\left\langle i_{0}, \ldots, i_{p}\right\rangle$ and $\alpha^{\prime \prime}$, corresponding to parameters $s_{p+1}, \ldots, s_{r-1}$, runs from $\left\langle i_{p}\right\rangle$ to $\left\langle i_{r}\right\rangle$ in $\left\langle i_{p}, \ldots, i_{r}\right\rangle$. Parallel transport along $\alpha^{\prime}$ takes $\mathbf{e}_{i_{0}}$ to $V_{\left\langle i_{0}, \ldots, i_{p}\right\rangle}\left(s_{1}, \ldots, s_{p-1}\right) \cdot \mathbf{e}_{i_{p}}$; parallel transport along $\alpha^{\prime \prime}$ takes $\mathbf{e}_{i_{p}}$ to $V_{\left\langle i_{p}, \ldots, i_{r}\right\rangle}\left(s_{p+1}, \ldots, s_{r-1}\right) \cdot \mathbf{e}_{i_{r}}$; the compatibility condition then follows from $G$-equivariance of parallel transport.

f) There is a widely shared intuition that the system of transition functions that makes up a coordinate bundle, in the terminology of Steenrod [26], is itself a "connection." Formally, it is a Cech 1-cocycle on the base space with values in the structure group, and can be interpreted as the macroscopic form of a connection (which is locally a 1 -form with values in the Lie Algebra of that group). A p.t.f., 


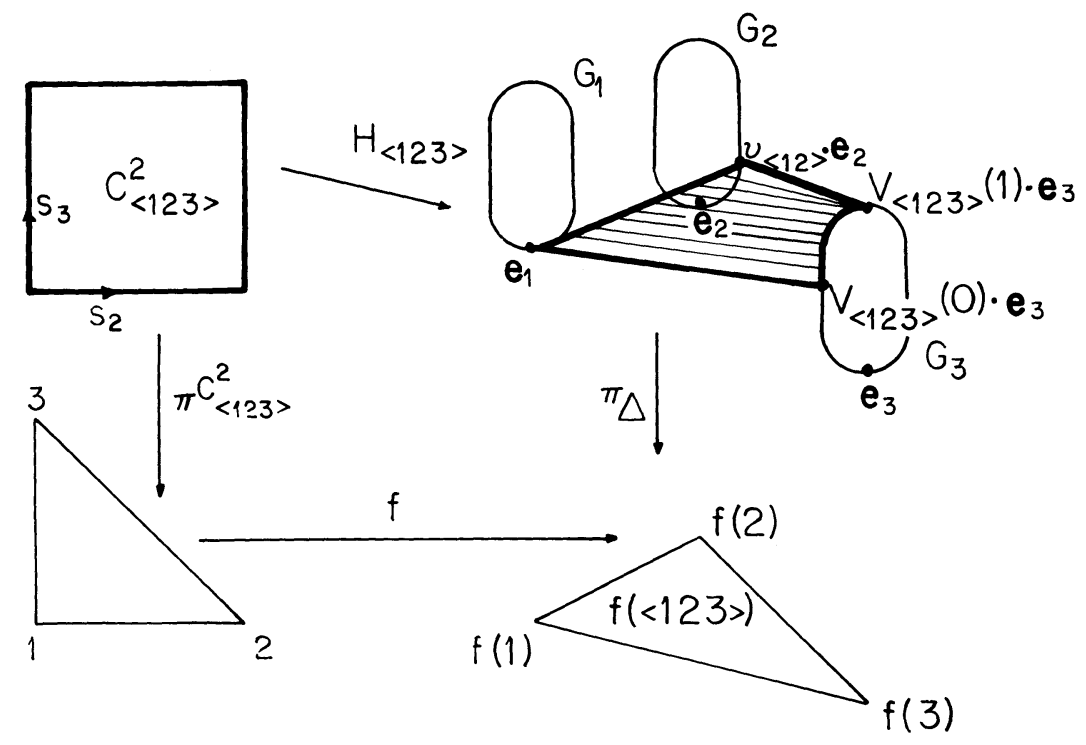

Fig. 2. Over the simplex $\langle 123\rangle$, the canonical pseudosection is built up from the maps $V_{\langle 12\rangle}: c_{\langle 12\rangle}^{0} \rightarrow G$ and $V_{\langle 123\rangle}: C_{\langle 123\rangle}^{1} \rightarrow G$. Here $G$ is drawn as a circle

in view of items c) and e) above, gives a different expression of this conceptual equivalence.

g) Concerning global geometry, let $G, \xi$ and $\omega$ be as in e) above; then the corresponding p.t.f. enables us to approximate the holonomy group of $\omega$. Roughly speaking, let $\mathscr{G}_{*}$ be as in d) above, and let $\mathscr{F}\left(\Lambda_{*}\right)$ be the cobar construction on $\Lambda$ (See [2]; this is a chain-complex model of the loop space $\Omega(|\Lambda|)$ ); then the p.t.f. determines a homomorphism of algebras $\mathscr{F}\left(\Lambda_{*}\right) \rightarrow \mathscr{G}_{*}$ which is a model for the holonomy map $\Omega(|\Lambda|) \rightarrow G$ of $\omega$.

A p.t.f. enters into our proof and calculations via its canonical pseudosection. This is defined as follows, using Milnor's model of the universal $G$-bundle (see 1.7).

Definition 2.3. The canonical pseudosection $\mathbf{H}$ of a parallel transport function $\mathbf{V}$ defined on a simplicial complex $\Lambda$ (see Fig. 2) is a collection of maps $H_{\sigma}: C_{\sigma}^{r} \rightarrow E_{\Delta} G$, one for each $\sigma \in \Lambda(r=\operatorname{dim} \sigma)$, satisfying

1. if $\sigma=\langle i\rangle, H_{\sigma}: C_{\sigma}^{0} \rightarrow E_{\Delta} G$ takes $\overrightarrow{0}$ to $\mathbf{e}_{i}$, the identity in $G_{i}$.

2. $H_{\left\langle i_{0}, \ldots, i_{r}\right\rangle}\left(s_{i_{1}}, \ldots, s_{i_{r}}\right)=\left(1-s_{i_{r}}\right) H_{\left\langle i_{0}, \ldots, i_{r-1}\right\rangle}\left(s_{i_{1}}, \ldots, s_{i_{r-1}}\right)$

$$
+s_{i_{r}} V_{\left\langle i_{0}, \ldots, i_{r}\right\rangle}\left(s_{i_{1}}, \ldots, s_{i_{r-1}}\right) \mathbf{e}_{i_{r}} \text {, }
$$

where $\mathbf{e}_{j}$ is the identity element in $G_{j}$.

These two conditions permit an inductive definition of $\mathbf{H}$, beginning with the 0 -simplexes of $\Lambda$. In addition the $H_{\sigma}$ 's clearly will satisfy

3. $\pi^{\infty} \circ H_{\sigma}=\pi^{r_{\sigma}^{r}}: C_{\sigma}^{r} \rightarrow \sigma$,

where $\pi^{\infty}: E_{\Delta} G \rightarrow \Delta^{\infty}$ is the projection defined in 1.7 and $\pi^{C_{\sigma}^{r}}$ is the standard projection of 1.6. Here we are using the identification of $\Lambda$ with a subcomplex of $\Delta^{\infty}$, so $\sigma \subset \Lambda^{\infty}$. 
Proposition 2.4. Let $\mathbf{V}$ be a G-valued parallel transport function on the ordered simplicial complex $\Lambda$, and $\mathbf{H}$ the canonical pseudosection of $\mathbf{V}$. Given $x \in \sigma \in \Lambda$, with $\operatorname{dim} \sigma=r$, choose $y \in\left(\pi^{C_{\sigma}^{r}}\right)^{-1}(x)$ and set $f_{\sigma}(x)=\pi_{\Delta}\left(H_{\sigma}(y)\right)$. Then

a) this gives a well defined map $f_{\sigma}:|\sigma| \rightarrow B_{\Delta} G$;

b) the various $f_{\sigma}$ 's fit together to give a well defined map $f: X \rightarrow B_{\Delta} G$. Thus $\mathbf{V}$ determines the bundle $\xi=f^{-1} \gamma_{\Delta} G$.

Proof of a). Consider two points $y, y^{\prime}$ with $\pi^{c_{\sigma}^{r}}(y)=\pi^{c^{r}}\left(y^{\prime}\right)$. For simplicity of notation let us suppose again here that $\sigma=\langle 0,1, \ldots, r\rangle$. Examination of the map $\pi^{c_{\sigma}^{r}}$ shows that either $y$ and $y^{\prime}$ both have $s_{r}=1$, or they have the same $s_{r}$-value and $s_{r-1}=1$, or ..., or they have the same $s_{r}, s_{r-1}, \ldots, s_{3}$-values and $s_{2}=1$, or all their coordinates are the same. In the first case formula 2) shows $H_{\sigma}(y)=V_{\sigma}\left(s_{1}, \ldots, s_{r-1}\right) \mathbf{e}_{r}$ and $H_{\sigma}\left(y^{\prime}\right)=V_{\sigma}\left(s_{1}^{\prime}, \ldots, s_{r-1}^{\prime}\right) \mathbf{e}_{r}$. These two points of $E_{\Delta} G$ are clearly $G$-equivalent.

In the second case, formula 2) gives (with notation from Sect. 1)

$$
\begin{aligned}
& H_{\sigma}(y)=\left(1-s_{r}\right) H_{\langle 0, \ldots, r-1\rangle}\left(s_{1}, \ldots, s_{r-2}, 1\right)+s_{r} V_{\sigma}\left(s_{1}, \ldots, s_{r-2}, 1\right) \mathbf{e}_{r}, \\
& H_{\sigma}\left(y^{\prime}\right)=\left(1-s_{r}\right) H_{\langle 0, \ldots, r-1\rangle}\left(s_{1}^{\prime}, \ldots, s_{r-2}^{\prime}, 1\right)+s_{r} V_{\sigma}\left(s_{1}^{\prime}, \ldots, s_{r-2}^{\prime}, 1\right) \mathbf{e}_{r} .
\end{aligned}
$$

Let us unravel the formulas, using

$$
\begin{aligned}
H_{\langle 0, \ldots, r-1\rangle}\left(s_{1}, \ldots, s_{r-2}, 1\right) & =V_{\langle 0, \ldots, r-1\rangle}\left(s_{1}, \ldots, s_{r-2}\right), \\
H_{\langle 0, \ldots, r-1\rangle}\left(s_{1}^{\prime}, \ldots, s_{r-2}^{\prime}, 1\right) & =V_{\langle 0, \ldots, r-1\rangle}\left(s_{1}^{\prime}, \ldots, s_{r-2}^{\prime}\right), \\
V_{\sigma}\left(s_{1}, \ldots, s_{r-2}, 1\right) & =V_{\langle 0, \ldots, r-1\rangle}\left(s_{1}, \ldots, s_{r-2}\right) \cdot v_{\langle r-1, r\rangle}, \\
V_{\sigma}\left(s_{1}^{\prime}, \ldots, s_{r-2}^{\prime}, 1\right) & =V_{\langle 0, \ldots, r-1\rangle}\left(s_{1}^{\prime}, \ldots, s_{r-2}^{\prime}\right) \cdot v_{\langle r-1, r\rangle} .
\end{aligned}
$$

It is now clear that $H_{\sigma}(y)=g \cdot H_{\sigma}\left(y^{\prime}\right)$, where

$$
g=V_{\langle 0, \ldots, r-1\rangle}\left(s_{1}, \ldots, s_{r-2}\right) \cdot\left(V_{\langle 0, \ldots, r-1\rangle}\left(s_{1}^{\prime}, \ldots, s_{r-2}^{\prime}\right)\right)^{-1} .
$$

In the next-to-last case mentioned,

$$
\begin{aligned}
H_{\sigma}(y)= & \left(1-s_{r}\right) H_{\langle 0, \ldots, r-1\rangle}\left(s_{1}, 1, s_{3}, \ldots, s_{r-2}, s_{r-1}\right) \\
& +s_{r} V_{\sigma}\left(s_{1}, 1, s_{3}, \ldots, s_{r-2}, s_{r-1}\right) \mathbf{e}_{r} \\
= & \left(1-s_{r}\right)\left[\left(1-s_{r-1}\right) H_{\langle 0, \ldots, r-2\rangle}\left(s_{1}, 1, s_{3}, \ldots, s_{r-2}\right)\right. \\
& \left.+s_{r-1} V_{\langle 0, \ldots, r-1\rangle}\left(s_{1}, 1, s_{3}, \ldots, s_{r-2}\right) \mathbf{e}_{r-1}\right] \\
& +s_{r} V_{\sigma}\left(s_{1}, 1, s_{3}, \ldots, s_{r-2}, s_{r-1}\right) \mathbf{e}_{r} \\
= & s_{r} V_{\sigma}\left(s_{1}, 1, s_{3}, \ldots, s_{r-2}, s_{r-1}\right) \mathbf{e}_{r} \\
& +\left(1-s_{r}\right) s_{r-1} V_{\langle 0, \ldots, r-1\rangle}\left(s_{1}, 1, s_{3}, \ldots, s_{r-2}\right) \mathbf{e}_{r-1} \\
& +\ldots \\
& +\left(1-s_{r}\right) \ldots\left(1-s_{3}\right) V_{\langle 012\rangle}\left(s_{1}\right) \mathbf{e}_{2}
\end{aligned}
$$

and $H_{\sigma}\left(y^{\prime}\right)$ has the same expression, except that the coordinate $s_{1}$ is replaced by $s_{1}^{\prime}$. So the two $H_{\sigma}$-values are related as above, with $g=V_{\langle 012\rangle}\left(s_{1}\right) .\left(V_{\langle 012\rangle}\left(s_{1}^{\prime}\right)\right)^{-1}$. Intermediate cases correspond to intermediate formulas for $g$, and in the last case $y=y^{\prime}$ so there is nothing to prove. 
Proof of b). Consider the case of $\tau^{k} \prec \sigma^{r}$, and of a point $x \in|\tau|$. We need to show that $f_{\sigma}(x)=f_{\tau}(x)$, i.e. that if $y \in C_{\tau}^{k}$ with $\pi^{c_{\tau}^{k}}(y)=(x)$ and $y^{\prime} \in C_{\sigma}^{r}$ with $\pi^{C_{\sigma}^{r}}\left(y^{\prime}\right)=(x)$, then there exists $g \in G$ with $H_{\tau}(y)=g \cdot H_{\sigma}\left(y^{\prime}\right)$.

It is sufficient by iteration to show this when $\tau$ is an $(r-1)$-face of $\sigma$. As before, suppose $\sigma=\langle 0,1, \ldots, r\rangle$. There are two cases to consider, according as $\tau$ does or does not contain the vertex $\langle 0\rangle$.

If $\langle 0\rangle \in \tau$ then $C_{\tau}^{r-1}$ is a face of $C_{\sigma}^{r}$, and $H_{\tau}(y)=\left.H_{\sigma}\right|_{C_{\tau}^{r-1}}(y)$, so the claim follows from part a). Otherwise, $\tau=\langle 1,2, \ldots, r\rangle$. We will prove by induction on $r$ that $H_{\tau}\left(s_{2}, \ldots, s_{r}\right)=v_{\langle 01\rangle}^{-1} \cdot H_{\sigma}\left(1, s_{2}, \ldots, s_{r}\right)$. Since $\pi^{C_{\sigma}^{r}}\left(1, s_{2}, \ldots, s_{r}\right)$ is also equal to $x$, the claim will again follow from part a).

We begin with $r=2$.

$$
\begin{aligned}
H_{\langle 012\rangle}\left(1, s_{2}\right) & =\left(1-s_{2}\right) H_{\langle 01\rangle}(1)+s_{2} V_{\langle 012\rangle}(1) \mathbf{e}_{2} \\
& =\left(1-s_{2}\right) v_{\langle 01\rangle} \mathbf{e}_{1}+s_{2} v_{\langle 01\rangle} v_{\langle 12\rangle} \mathbf{e}_{2} \\
& =v_{\langle 01\rangle}\left[\left(1-s_{2}\right) \mathbf{e}_{1}+s_{1} v_{\langle 12\rangle} \mathbf{e}_{2}\right] \\
& =v_{\langle 01\rangle} H_{\langle 12\rangle}\left(s_{2}\right) .
\end{aligned}
$$

Suppose the identity has been proved for $r-1$. Then

$$
H_{\langle 01, \ldots, r-1\rangle}\left(1, s_{2}, \ldots, s_{r-1}\right)=v_{\langle 01\rangle} H_{\langle 1, \ldots, r-1\rangle}\left(s_{2}, \ldots, s_{r-1}\right) .
$$

This gives

$$
\begin{aligned}
H_{\sigma}\left(1, s_{2}, \ldots, s_{r}\right)= & \left(1-s_{r}\right) v_{\langle 01\rangle} H_{\langle 1, \ldots, r-1\rangle}\left(s_{2}, \ldots, s_{r-1}\right)+s_{r} V_{\sigma}\left(1, s_{2}, \ldots, s_{r}\right) \cdot \mathbf{e}_{r} \\
= & v_{\langle 01\rangle}\left[\left(1-s_{r}\right) H_{\langle 1, \ldots, r-1\rangle}\left(s_{2}, \ldots, s_{r-1}\right)\right. \\
& \left.+s_{r} V_{\langle 1, \ldots, r\rangle}\left(s_{2}, \ldots, s_{r-1}\right) \cdot \mathbf{e}_{r}\right] \\
= & v_{\langle 01\rangle} H_{\tau}\left(s_{2}, \ldots, s_{r}\right),
\end{aligned}
$$

which completes the proof of the proposition.

2.5 Remark. The map $f$ embeds $X$ in $B_{\Delta} G$. This follows from the fact that the composition $\pi^{\infty} \circ f: X \rightarrow \Delta^{\infty}$ (where $\pi^{\infty}: B_{\Delta} G \rightarrow \Delta^{\infty}$ is as in 1.7) maps each simplex of $\Lambda$ homeomorphically onto the corresponding simplex of $\Delta^{\infty}$. Consequently $\xi=\gamma_{\Delta} G \mid f(X)$. This allows us to consider $E$ as a subset of $E_{\Delta} G$, and the $H_{\sigma}$ 's as maps into $E$; it is in this sense that $\mathbf{H}$ is a pseudosection.

\section{Lattice Gauge Fields}

3.1. In [24] we showed that a $S U(2)$-valued lattice gauge field satisfying a certain nonsingularity condition could be embedded in a $S U(2)$-coordinate bundle; and we gave an algorithm for computing the second Chern class of that bundle directly from the lattice data. Here we are interested in $G L(p, \mathbf{C})$-valued lattice gauge fields, but for this group the convenient spherical geometry of $S U(2)$ is no longer available. Our substitute is to think of $G L(p, \mathbf{C})$ as sitting inside $M(p)$, the affine space of all $p \times p$ complex matrices, and to use the affine geometry of $M(p)$ to do the necessary interpolations to make a lattice gauge field $\mathbf{u}$ into a more continuous object. Clearly, 
the closer the transporters $u_{i j}$ lie to the set of singular matrices in $M(p)$, the less room there is for these interpolations. We assign to $\mathbf{u}$ a distortion coefficient $K(\mathbf{u})$ (this coefficient is 1 , for example, if the transporters are all unitary), and give an algorithm which, if the modulus of continuity of $\mathbf{u}$ is sufficiently small with respect to $K(\mathbf{u})$, will produce from $\mathbf{u}$ a $G L(p, \mathbf{C})$-valued parallel transport function. We will also show that under a more stringent condition on the modulus of continuity, the bundle determined by that p.t.f. does not depend on the interpolation algorithm chosen, and may therefore be called the bundle determined by $\mathbf{u}$.

Definition 3.2. Given a Lie group $G$, a G-valued lattice gauge field (1.g.f.) $\mathbf{u}$ on a simplicial complex $\Lambda$ is a function that assigns to each 1 -simplex $\langle i j\rangle$ of $\Lambda$ an element $u_{i j} \in G$, subject to the condition $u_{j i}=\left(u_{i j}\right)^{-1}$.

3.3. Let $M(p)$ be the algebra of all $p \times p$ complex-valued matrices. For $A \in M(p)$ we take, as usual,

$$
\|A\|=\underset{|\vec{v}|=1}{\max }|A \vec{v}|
$$

and for any $\rho \geqq 0$ we let $B(A, \rho)$ denote the open ball of radius $\rho$ about $A$ in $M(p)$ :

$$
B(A, \rho)=\left\{A^{\prime}:\left\|A-A^{\prime}\right\|<\rho\right\} .
$$

Definition 3.4. For $A \in G L(p, \mathbf{C})$ we define the distortion coefficient of $A$ to be the number

$$
K(A)=\max \left\{\|A\|,\left\|A^{-1}\right\|\right\} .
$$

(Note that $K(A) \geqq 1$, with equality if and only if $A \in U(p)$.) The distortion coefficient of a l.g.f. u with values in $G L(p, \mathbf{C})$ is then

$$
K(\mathbf{u})=\max _{\langle i j\rangle \in \Lambda} K\left(u_{i j}\right) .
$$

Lemma 3.5. Let $A$ be an element of $G=G L(p, \mathbf{C})$ with distortion coefficient $K=K(A)$; then

$$
B(A, 1 / K) \subseteq G
$$

Proof. Suppose $A^{\prime} \in B(A, 1 / K)$ but $A^{\prime} \notin G$, so $A^{\prime} \vec{v}=0$ for some $\vec{v}$ with $|\vec{v}|=1$. Then $|A \vec{v}|=|A \vec{v}| /|\vec{v}|=|\vec{w}| /\left|A^{-1} \vec{w}\right| \geqq 1 / K$, with $\vec{w}=A \vec{v}$. On the other hand $|A \vec{v}|=\left|A \vec{v}-A^{\prime} \vec{v}\right| \leqq\left\|A-A^{\prime}\right\|<1 / K$, a contradiction.

Definition 3.6. Let $\Lambda$ be a simplicial complex with vertex ordering $\mathbf{0}$, and $\mathbf{u}$ a $G L(p, \mathbf{C})$-valued 1.g.f. on $\Lambda$. The modulus of continuity of $\mathbf{u}$ with respect to $\mathbf{o}$ or, more briefly, its o-modulus of continuity, is the number

$$
\delta=\max \left\|u_{i j} \cdot u_{j k}-u_{i k}\right\|,
$$

where the maximum is taken over the set of all 2-simplexes $\langle i j k\rangle$ (with vertices so o-ordered).

Definition 3.7. A l.g.f. $\mathbf{u}$ on $\Lambda$, as above, with distortion coefficient $K$ is continuous 
with respect to $\mathbf{0}$ if its $\mathbf{0}$-modulus of continuity $\delta$ satisfies

$$
\delta<\frac{1}{K \varphi(K, n)},
$$

where $n=\operatorname{dim} \Lambda$ and, for $K \geqq 1$ and $l=1,2, \ldots$,

$$
\varphi(K, l)=1+K+K^{2}+\cdots+K^{l-1} .
$$

(Note that $\varphi(K, l) \geqq l$ with equality if $K=1$ or $l=1$.)

Proposition 3.8. Let $\mathbf{u}$ be a $G L(p, \mathbf{C})$-valued l.g.f. on the $n$-dimensional locally ordered simplicial complex $\Lambda$, with distortion coefficient $K$ and $\mathbf{0}$-modulus of continuity $\delta$ satisfying $\delta<1 / K \varphi(K, n)$ (so $\mathbf{u}$ is continuous with respect to $\mathbf{0}$ ). Then there is a $G L(p, \mathbf{C})$-valued p.t.f. $\mathbf{V}$ on $\Lambda$ such that

(1) $v_{\langle i j\rangle}=u_{i j}$ whenever $\langle i j\rangle \in \Lambda$ with $i$ o-preceding $j$;

(2) For every $\sigma \in \Lambda$ with $\operatorname{dim} \sigma=r \geqq 2$, and every $\vec{s} \in c_{\sigma}^{r-1}$,

$$
\left\|V_{\sigma}(\vec{s})-u_{\sigma}\right\|<\delta \varphi(K, r) .
$$

(Here $u_{\sigma}=u_{i j}$, where $\langle i\rangle$ and $\langle j\rangle$ are the first- and last-ordered vertices of $\sigma$ ).

Proof. The construction of $V_{\sigma}$ is made by induction on $\operatorname{dim} \sigma$. When $\operatorname{dim} \sigma=1, V_{\sigma}$ is determined by (1).

In general, the compatibility conditions of Definition 2.1 determine $V_{\sigma}$ on the vertices of $c_{\sigma}$. More explicitly, setting $\sigma=\langle 0, \ldots, r\rangle$, every vertex of $c_{\sigma}^{r-1}$ can be uniquely determined by a subset $I \subset\{1, \ldots, r-1\}$ as follows: the subset $I=\left(i_{1}<\cdots<i_{k}\right)$ corresponds to the vertex $\sum_{j=1}^{k} \mathbf{e}_{i_{j}}$ (or to the origin $\overrightarrow{0}$ when $\left.I=\varnothing\right)$. Set $u_{I}=u_{0 i_{1}} \cdot u_{i_{1} i_{2}} \cdots u_{i_{r} r}$; then by the axioms for a p.t.f. (Definition 2.1), $V_{\sigma}\left(\Sigma \mathbf{e}_{i_{j}}\right)=u_{I}$. In particular, $V_{\sigma}(\overrightarrow{0})=u_{0 r}$.

We define $V_{\sigma}$ initially as a map $V_{\sigma}: c_{\sigma}^{r-1} \rightarrow M(p)$; then we shall prove that in fact $\operatorname{Im}\left(V_{\sigma}\right) \subset G L(p, \mathbf{C})$. The definition proceeds by successive linear interpolation in the coordinates $s_{1}, \ldots, s_{r-1}$. Specifically,

$$
\begin{aligned}
V_{\sigma}\left(s_{1}, \ldots, s_{r-1}\right)= & \left(1-s_{r-1}\right) V_{\langle 01, \ldots, r-2, r\rangle}\left(s_{1}, \ldots, s_{r-2}\right) \\
& +s_{r-1} V_{\langle 01, \ldots, r-1\rangle}\left(s_{1}, \ldots, s_{r-2}\right) \cdot u_{r-1, r} .
\end{aligned}
$$

We now prove by induction on $k$ that if $I=\left\{i_{1}, \ldots, i_{k}\right\}$ as above, then

$$
\text { (*) }\left\|u_{I}-u_{0 r}\right\|<\delta \varphi(K, k) .
$$

This is trivial when $I=\varnothing$, and follows from the continuity of $\mathbf{u}$ when $k=1$. For $k \geqq 2$ we have

$$
\begin{aligned}
u_{I}-u_{0 r}= & \left(u_{0 i_{1}} \cdot u_{i_{1} i_{2}}-u_{0 i_{2}}\right) \cdot\left(u_{i_{2} i_{3}} \cdots u_{i_{k} r}\right) \\
& +\left(u_{0 i_{2}} \cdot u_{i_{2} i_{3}} \cdots u_{i_{k} r}-u_{0 r}\right) .
\end{aligned}
$$

Using the inductive hypothesis and the fact that each $\left\|u_{i j}\right\| \leqq K$, it follows that

$$
\left\|u_{I}-u_{0 r}\right\|<\delta K^{k-1}+\delta \varphi(K, k-1)=\delta \varphi(K, k) .
$$

Thus (*) holds. 
In particular, assertion (2) in the statement of the proposition holds at every vertex of $c_{\sigma}^{r-1}$. Since the \|\| -balls are convex, the construction of $V_{\sigma}$ using successive linear interpolations now implies (2) in general. Since $\delta \varphi(K, k)<1 / K$, it follows from Lemma 3.5 that $V_{\sigma}$ actually takes values in $G$. To complete the proof that $\mathbf{V}=\left\{V_{\sigma}\right\}$ is a p.t.f., it remains to check the product axiom of 2.1. To do so, it is convenient to develop another formula for $V_{\sigma}$, which will also be useful in the sequel. For each $I$ as above, define the function $\lambda_{I}: c_{\sigma}^{r-1} \rightarrow[0,1]$ by

$$
\lambda_{I}\left(s_{1}, \ldots, s_{r-1}\right)=\prod_{i \in I} s_{i} \prod_{J \notin I}\left(1-s_{j}\right) .
$$

Then

$$
(* *) \quad V_{\sigma}\left(s_{1}, \ldots, s_{r-1}\right)=\sum_{I} \lambda_{I}\left(s_{1}, \ldots, s_{r-1}\right) u_{I} .
$$

The product axiom now follows.

It is natural to ask to what extent the bundle determined by $\mathbf{V}$ really depends on $\mathbf{u}$; our uniqueness result, stated below and proved in Sect. 6, will require a stricter bound on the modulus of continuity.

Definition 3.9. A $G$-valued l.g.f. u of distortion coefficient $K$ on an $n$-dimensional simplicial complex $\Lambda$ with local vertex ordering $\mathbf{o}$ is strictly continuous with respect to $\mathbf{0}$ if its $\mathbf{0}$-modulus of continuity $\delta$ satisfies

$$
\delta<\frac{1}{4 n K^{2} \varphi(K, n)} .
$$

Note that for a unitary 1.g.f. this collapses to $\delta<1 / 4 n^{2}$.

Proposition 3.10. Let $\mathbf{u}$ be a strictly continuous $G$-valued l.g.f. on the locally ordered simplicial complex $\Lambda$, with distortion coefficient $K$ and $\mathbf{0}$-modulus of continuity $\delta$, and let $\mathbf{V}$ and $\mathbf{V}^{\prime}$ be two $G$-valued p.t.f.'s on $\Lambda$ such that for every $\sigma$ of dimension $r \geqq 1$ and every $\vec{s} \in c_{\sigma}^{r-1}$, we have

$$
\begin{gathered}
\left\|V_{\sigma}(\vec{s})-u_{\sigma}\right\|<\delta \varphi(K, r), \\
\left\|V_{\sigma}^{\prime}(\vec{s})-u_{\sigma}\right\|<\delta \varphi(K, r)
\end{gathered}
$$

(for example, one could be the p.t.f. constructed from $\mathbf{u}$ by the method of Proposition 3.8). Then $\mathbf{V}$ and $\mathbf{V}^{\prime}$ determine the same bundle. We will call this the bundle determined by $\mathbf{u}$.

Definition 3.11. Let $\mathbf{V}$ and $\mathbf{V}^{\prime}$ be $G$-valued p.t.f.'s on $\Lambda$. Then $\mathbf{V}^{\prime}$ is an $\varepsilon$-approximation to $\mathbf{V}$ if

$$
\left\|V_{\sigma}^{\prime}(\vec{s})-V_{\sigma}(\vec{s})\right\|<\varepsilon
$$

for every $\sigma \in \Lambda$ and every $\vec{s} \in c_{\sigma}$.

Corollary 3.12. Let $\mathbf{u}$ be a G-valued l.g.f. on the locally ordered simplicial complex $\Lambda$ which is strictly continuous with respect to $\mathbf{0}$, and let $\mathbf{V}$ be a G-valued p.t.f. on $\Lambda$ satisfying

$$
\left\|V_{\sigma}(\vec{s})-u_{\sigma}\right\|<\delta \varphi(K, r)
$$


where $r=\operatorname{dim} \sigma$, for every $\sigma \in \Lambda$ and every $\vec{s} \in c_{\sigma}$. Then there exists $\varepsilon>0$ such that any p.t.f. $\mathbf{V}^{\prime}$ which is an E-approximation to $\mathbf{V}$ determines the same bundle as $\mathbf{V}$.

Proof. Let $\eta_{r}=\max \left\|V_{\sigma}(\vec{s})-u_{\sigma}\right\|$, where the maximum is taken over the compact set $\bigcup c_{\sigma}^{r-1}$, so $\eta_{r}<\delta \varphi(K, r)$. Choose a positive $\varepsilon<\min \left(\delta \varphi(K, r)-\eta_{r}\right)$. Then $\sigma \in \Lambda, \operatorname{dim} \sigma=r$
$\left\|V_{\sigma}^{\prime}(\vec{s})-V_{\sigma}(\vec{s})\right\|<\varepsilon$ implies for every $\sigma$ of dimension ${ }^{r} r$, and $\vec{s} \in c_{\sigma}^{r-1}$, that $\left\|V_{\sigma}^{\prime}(\vec{s})-u_{\sigma}\right\|<\varepsilon+\eta_{r}<\delta \varphi(K, r)$; we may now apply Proposition 3.10.

Definition 3.13. Let $\mathbf{u}$ and $\mathbf{u}^{\prime}$ be $G$-valued l.g.f.'s on the simplicial complex $\Lambda$. We will say $\mathbf{u}^{\prime}$ is an $\varepsilon$-approximation to $\mathbf{u}$ if

$$
\left\|u_{i j}-u_{i j}^{\prime}\right\|<\varepsilon
$$

for every $\langle i j\rangle \in \Lambda$.

Corollary 3.14. Let $\mathbf{u}^{0}$ be a strictly continuous G-valued 1.g.f. on the locally ordered simplicial complex $\Lambda$. Then there exists $\varepsilon>0$ such that if $\mathbf{u}$ is an $\varepsilon$-approximation to $\mathbf{u}^{0}$, then $\mathbf{u}$ is strictly continuous and determines the same bundle as $\mathbf{u}^{0}$.

Proof. Let $K^{0}$ and $\delta^{0}$ be the distortion coefficient and modulus of continuity of $\mathbf{u}^{0}$. Strict continuity of $\mathbf{u}^{0}$ means $\delta^{0}<1 / 4 n\left(K^{0}\right)^{2} \varphi\left(K^{0}, n\right)$. Choose $\delta$ and $\alpha>1$ such that

$$
\delta^{0}<\delta<\frac{1}{4 n\left(\alpha K^{0}\right)^{2} \varphi\left(\alpha K^{0}, n\right)}<\frac{1}{4 n\left(K^{0}\right)^{2} \varphi\left(K^{0}, n\right)} .
$$

A first condition on $\varepsilon$ is to choose it so that

$$
K(\mathbf{u})<\alpha K^{0}, \quad \delta(\mathbf{u})<\delta
$$

for any $\mathbf{u}$ which is an $\varepsilon$-approximation to $\mathbf{u}^{0}$; such a $\mathbf{u}$ will also be strictly continuous. The rest of the proof follows immediately from Corollary 3.12 and formula $(* *)$ in the proof of Proposition 3.8.

\section{Local Intersection-Theoretic Computation of Characteristic Classes of Lattice Gauge Fields}

We shall now show that, provided the lattice gauge field $\mathbf{u}$ is "generic," the Chern classes of the bundle $\xi$ determined by $\mathbf{V}$, where $\mathbf{V}$ is the parallel transport function defined from $\mathbf{u}$ in Proposition 3.8, can be evaluated from $\mathbf{u}$ in terms of intersection numbers between explicit, polynomially defined geometric cycles in the linear space $M(p)$ of all $p \times p$ complex-valued matrices. We continue with the notation $G=G L(p, \mathbf{C})$.

Let $\Lambda$ be a locally ordered, finite simplicial complex, and let $\mathbf{V}$ be a $G$-valued p.t.f. on $\Lambda$, with canonical pseudosection $\mathbf{H}$. Let $f: X \rightarrow B_{\Delta} G$ be the classifying map constructed from $\mathbf{H}$ in Proposition 2.4 (where $X=|\Lambda|), \xi=(\pi: E \rightarrow X$ ) the corresponding $G$-bundle and $\tilde{f}: E \rightarrow E_{\Delta} G$ the bundle map covering $f$.

4.1. Let $m: G \rightarrow M(p)$ be the inclusion map. We extend $m$ to a map $M_{\Delta}: E_{\Delta} G \rightarrow M(p)$ by

$$
M_{\Delta}\left(\sum t_{i} g_{i} \mathbf{e}_{i}\right)=\sum t_{i} m\left(g_{i}\right)
$$


In addition, for each $\sigma \in \Lambda$, let $M_{\sigma}: C_{\sigma}^{r} \rightarrow M(p)$ be given by

$$
M_{\sigma}=M_{\Delta} \circ H_{\sigma} .
$$

4.2. In case $\mathbf{V}$ is defined from a continuous lattice gauge field $\mathbf{u}$, we can give an explicit formula for $M_{\sigma}$ in terms of $\mathbf{u}$. Say $\sigma=\langle 0, \ldots, r\rangle$, with vertices ordered as listed. Let $I$ be any subset of $\{1, \ldots, r\}$; say $I=\left(i_{1}<\cdots<i_{k}\right)$. Set $\tilde{u}_{I}=u_{0 i_{1}} \cdots u_{i_{k-1} i_{k}}$ (with the convention $\tilde{u}_{\varnothing}=\mathbf{1}$, the identity matrix). With $\lambda_{I}$ defined as in the proof of Proposition 3.8, we have

$$
M_{\sigma}\left(s_{1}, \ldots, s_{r}\right)=\sum_{I} \lambda_{I}\left(s_{1}, \ldots, s_{r}\right) \tilde{u}_{I}
$$

This follows by a straightforward induction argument from Definition 2.3 and equation $(* *)$ in the proof of Proposition 3.8, where $u_{I}=\tilde{u}_{I} \cdot u_{i_{k}}$.

4.3. For any matrix $A \in M(p)$, let $A^{1}, \ldots, A^{p}$ be its column vectors. Define subsets $\Sigma_{q} \subseteq M(p)$, for $q=1, \ldots, p$ by

$$
\Sigma_{q}=\left\{A \mid \operatorname{rank}\left(A^{1}, \ldots, A^{p-q+1}\right) \leqq p-q\right\} .
$$

Thus $\Sigma_{1}=\{A \mid \operatorname{det} A=0\}$ and $\Sigma_{p}=\left\{A \mid A^{1}=0\right\}$. In general, setting $N=\left(\begin{array}{c}p \\ p-q+1\end{array}\right)$, $\Sigma_{p}$ is defined by the vanishing of the $N$ determinants $\varphi_{1}, \ldots, \varphi_{N}$ formed from $A^{1}, \ldots, A^{p-q+1}$, and is thus an algebraic variety. As such, it is a cycle with a canonical orientation (see, for example, [9, Chapter 0, Sect. 4]). Actually $\Sigma_{q}$ has real codimension $2 q$, so no more than $q$ of these $N$ determinant functions can be linearly independent at a point of $\Sigma_{q}$; this fact will be established during the proof of Theorem 4.8. For future reference it will be useful to study the structure of this set of equations a little more closely.

4.4. For a $p \times p$ matrix $A$, let $A^{\prime}$ be the $p \times(p-q+1)$ matrix constituted by its first $p-q+1$ columns, and let $U_{q} \subset \Sigma_{q}$ be the set of $A$ 's in $\Sigma_{q}$ such that any set of $p-q$ different rows of $A^{\prime}$ is linearly independent. Clearly $U_{q}$ is an open, dense subset of $\Sigma_{q}$. Now let $\varphi_{i_{1}}, \ldots, \varphi_{i_{q}}$ be any collection of the $\varphi$ 's satisfying the following independence contion: each $\varphi_{i_{j}}$ involves a row which does not appear in any of the others. (There are clearly at most $q$ determinants in such a collection.) Then it is straightforward to show that at any point $A$ of $U_{q}$ the $q$ gradients (in the sense of [20]) $\nabla \varphi_{i_{1}}, \ldots, \nabla \varphi_{i_{q}}$ are linearly independent. It follows that, near $A$, the variety $\Sigma_{q}$ is defined by the vanishing of $\varphi_{i_{1}}, \ldots, \varphi_{i_{q}}$.

We want to show that the Chern classes $c_{q}(\xi)$ of $\xi$ may be computed as $2 q$-cocycles on $\Lambda$ by means of the intersection numbers $M_{\sigma} \cdot \Sigma_{q}$, for $\operatorname{dim} \sigma=2 q$. But such intersection numbers are only defined for "generic" $\mathbf{V}$, and we must first explain this point.

Definition 4.5. A $G$-valued p.t.f. $\mathbf{V}$ on $\Lambda$ is generic with respect to $\left\{\Sigma_{q}\right\}$ if

(1) Each $V_{\sigma}: c_{\sigma} \rightarrow G$ is piecewise differentiable.

(2) If $\operatorname{dim} \sigma=2 q$, then there are (none or) finitely many interior points $p_{1}, \ldots, p_{s} \in C_{\sigma}$ 
such that:

(i) the $2 q$-chain $M_{\sigma}$ meets $\Sigma_{q}$ in the points $M_{\sigma}\left(p_{i}\right)$, which belong to $U_{q}$;

(ii) $M_{\sigma}$ is differentiable at each $p_{i}$;

(iii) $M_{\sigma}$ and $\Sigma_{q}$ are transverse at each $M_{\sigma}\left(p_{i}\right)$ (in particular, if $\operatorname{dim} \sigma<2 q$, then $\left.M_{\sigma} \cap \Sigma_{q}=\varnothing\right)$.

Definition 4.6. Let $\mathbf{u}$ be a $G$-valued l.g.f. on $\Lambda$ which is continuous with respect to $\mathbf{0}$, and let $\mathbf{V}$ be the p.t.f. constructed from $\mathbf{u}$ following the proof of Proposition 3.8; we say that $\mathbf{u}$ is generic if $\mathbf{V}$ is.

The following proposition is proved in Sect. 6.

Proposition 4.7. Let $\mathbf{u}^{0}$ be a strictly continuous G-valued l.g.f. on the locally ordered simplicial complex $\Lambda$. Then there exists an open neighborhood $U$ of $\mathbf{u}^{0}$ in the space of all $G$-valued l.g.f.'s on $\Lambda$ and in $U$ an open dense set $U^{\prime}$ consisting of l.g.f.'s which are generic and strictly continuous and which determine the same bundle as $\mathbf{u}^{0}$.

Theorem 4.8. Let $\mathbf{V}$ be a generic G-valued p.t.f. on a finite simplicial complex $\Lambda$ with local vertex ordering $\mathbf{0}$. Then, for $q=1, \ldots, p$, the $q^{\text {th }}$ Chern class $c_{q}(\xi)$ of the bundle $\xi$ associated to $\mathbf{V}$ by Proposition 2.4 is represented by the $2 q$-cocycle $C_{q}(\mathbf{V})$ on $\Lambda$ defined by

$$
C_{q}(\mathbf{V})(\sigma)=M_{\sigma}: \Sigma_{q}, \quad \operatorname{dim} \sigma=2 q .
$$

Here $M_{\sigma} \cdot \Sigma_{q}$ represents the intersection number $\Sigma_{i} N\left(M_{\sigma}, \Sigma_{q}, p_{i}\right)$, where the sum is taken over all the intersection points, and $N\left(M_{\sigma}, \Sigma_{q}, p_{i}\right)= \pm 1$ is determined as follows. Suppose $\sigma=\langle 0,1, \ldots, r\rangle$, with vertices so ordered, with $r=2 q . M(p)$ and $\Sigma_{q}$ both have canonical orientations, since one is a complex manifold and the other a complex subvariety. If the $2 q$-frame $\left(M_{\sigma}\right)_{*}\left(\partial / \partial s_{1}\right), \ldots,\left(M_{\sigma}\right)_{*}\left(\partial / \partial s_{r}\right)$, followed by a $2\left(p^{2}-q\right)$-frame giving the canonical orientation of $\Sigma_{q}$ gives a positively oriented $2 p^{2}$-frame (with respect to the canonical orientation of $M(p)$, then $N\left(M_{\sigma}, \Sigma_{q}, p_{i}\right)=+1$; otherwise it is -1 .

Proof. We start by embedding the local ordering $\mathbf{o}$ in a total ordering $1, \ldots, v$ (which we shall also call o) of the vertices of $\Lambda$. Set $E_{\Delta}^{v} G=G_{1} * \cdots * G_{v} \subseteq E_{\Delta} G$, $B_{\Delta}^{\nu} G=E_{\Delta}^{\nu} G / G \subseteq B_{\Delta} G$, and $\gamma_{\Delta}^{\nu}=\left(\pi_{\Delta}^{\nu}: E_{\Delta}^{\nu} G \rightarrow B_{\Delta}^{\nu} G\right)$ the principal $G$-bundle so defined. The classifying map $f: X \rightarrow B_{\Delta} G$ given in the proof of Proposition 2.4 has image contained in $B_{\Delta}^{v} G$, so we may regard $f$ as a map from $X=|\Lambda|$ into $B_{\Delta}^{v} G$. Now let $\gamma^{v} G=\left(\pi^{v}: E^{v} G \rightarrow B^{v} G\right)$ be the $G$-bundle of $p$-frames in $\mathbf{C}^{v p}$. We shall construct a $G$-equivariant map $\widetilde{\Psi}: E_{\Delta}^{v} G \rightarrow E^{v} G$; this will induce a map $\Psi: B_{\Delta}^{v} G \rightarrow B^{v} G$, and we will have $\xi=(\Psi \circ f)^{-1} \gamma^{\nu} G$.

To construct $\widetilde{\Psi}$ we number the coordinates in $\mathbf{C}^{v p} p$ by $p$, as follows: $z_{11}, \ldots, z_{1 p}, z_{21}, \ldots, z_{2 p}, \ldots, z_{v 1}, \ldots, z_{v p}$. Consider the point $g_{i} \mathbf{e}_{i} \in E_{\Delta}^{v} G$. Using matrix notation for $G=G L(p, \mathbf{C})$, let $g_{i, 1}, \ldots, g_{i, p}$ be the rows of $g_{i}$; then let $\tilde{\psi}_{1}\left(g_{i} \mathbf{e}_{i}\right) \in \mathbf{C}^{v p}$ be the vector with the entries of the row $g_{i, 1}$ in positions numbered $i 1$ through $i p$, and zeroes elsewhere; similarly $\tilde{\psi}_{2}\left(g_{i} \mathbf{e}_{i}\right)$ has the entries of $g_{i, 2}$ in positions numbered $i 1$ through $i p$, and zeroes elsewhere, etc. Finally let $\widetilde{\boldsymbol{\Psi}}\left(g_{i} \mathbf{e}_{i}\right)$ be the frame $\widetilde{\psi}_{1}\left(g_{i} \mathbf{e}_{i}\right), \ldots, \tilde{\psi}_{p}\left(g_{i} \mathbf{e}_{i}\right)$. Now extend each $\widetilde{\psi}_{j}$ linearly by

$$
\tilde{\psi}_{j}\left(\sum_{i} t_{i} g_{i} \mathbf{e}_{i}\right)=\sum_{i} t_{i} \tilde{\psi}_{j}\left(g_{i} \mathbf{e}_{i}\right),
$$


and define $\tilde{\Psi}\left(\sum_{i} t_{i} g_{i} \mathbf{e}_{i}\right)$ to be the ordered set of vectors $\tilde{\psi}_{1}\left(\sum_{i} t_{i} g_{i} \mathbf{e}_{i}\right), \ldots$, $\tilde{\psi}_{p}\left(\sum_{i} t_{i} g_{i} \mathbf{e}_{i}\right)$. This is in fact a frame, because at least one $t_{j}$ must be nonzero, and then linear independence can be checked using the components numbered $j 1, \ldots, j p$, which are just $t_{j}$ times those of the nonsingular matrix $g_{j}$.

If we identify $E^{v} G$ with the space of $p \times v p$ matrices (by using the standard basis corresponding to the $z_{i j}$ 's to associate to each vector the row-vector of its components, and by stacking these $p$ rows into a matrix) then two frames span the same $p$-plane if and only if they differ by left multiplication by some $g \in G$. The map $\tilde{\Psi}$ is clearly $G$-equivariant with respect to this multiplication and the left action of $G$ on $E_{\Delta}^{v} G$, and so induces $\Psi: B_{\Delta}^{v} G \rightarrow B^{v} G$, as promised.

The homology of $B^{v} G$ may be represented by Schubert cycles $[6,9$, Chapter 1 , Sect. 5]. These are defined as follows: choose a basis $\mathscr{F}=\left(f_{11}, \ldots, f_{1 p}, f_{21}, \ldots\right)$ for $C^{v p}$ and define $S_{q} \subset B^{v} G$ to be the set of $p$-planes whose projection into the span of $f_{11}, \ldots, f_{1, p-q+1}$ has dimension $\leqq p-q$. (This is the same as requiring, as in [6], that their intersection with the span of $f_{1, p-q+2}, \ldots, f_{v p}$ have dimension $\geqq q$.) This set has codimension $2 q$. If we use the basis $\mathscr{F}$ to identify each vector in the frame with a row vector, and the entire frame with a $p \times v p$ matrix, then the frame with matrix $F$ belongs to

$$
\text { (*) } \quad \tilde{S}_{q}=\left(\pi^{v}\right)^{-1} S_{q}
$$

if and only if rank $\left(F^{11}, \ldots, F^{1, p-q+1}\right) \leqq p-q$, where $F^{11}, F^{12}, \ldots$ are the column vectors of $F$. Now if $T: \mathbf{C}^{v p} \rightarrow \mathbf{C}^{p}$ is defined by projection onto the span of $f_{11}, \ldots, f_{1 p}$, then $T$ induces $\tilde{T}: E^{v} G \rightarrow M(p)$ which clearly satisfies

$$
\text { (**) } \quad \tilde{S}_{q}=\tilde{T}^{-1} \Sigma_{q} .
$$

(Comparing $(*)$ and $(* *)$ shows immediately that $\Sigma_{q}$ and $S_{q}$ have the same codimension, and that therefore $\Sigma_{q}$ is a variety of codimension $2 q$ as we claimed earlier.)

By naturality of the Chern classes, $c_{q}(\xi)=(\Psi \circ f)^{*} c_{q}\left(\gamma^{v} G\right)$. Since $c_{q}\left(\gamma^{v} G\right)$ is Poincaré dual to the homology class of $S_{q}[6]$, this means that $c_{q}(\xi)$ is represented by the $2 q$-cycle $C_{q}(\mathbf{V})(\sigma)=\Psi \circ f(\sigma) \cdot S_{q}$ (provided this intersection number is well defined).

For our purposes it is convenient to use the basis

$$
\begin{aligned}
& f_{11}=e_{11}, \quad \cdots \quad f_{1 p}=e_{1 p} \\
& f_{21}=e_{11}-e_{21}, \quad \cdots \quad f_{2 p}=e_{1 p}-e_{2 p} \\
& f_{v 1}=e_{11}-e_{v 1}, \quad \cdots \quad f_{v p}=e_{1 p}-e_{v p}
\end{aligned}
$$

where the $e_{i j}$ are the elements of the standard basis with respect to the coordinates $z_{i j}$ mentioned above.

Then if a vector has components $a_{11}, \ldots, a_{v p}$ with respect to the standard basis, its components with respect to $\mathscr{F}$ will be $b_{1 k}=a_{1 k}+a_{2 k}+\cdots+a_{v k}, b_{2 k}=$ 
$-a_{2 k}, \ldots, b_{v k}=-a_{v k}$, for $k=1, \ldots, p$. In particular,

$$
\tilde{T} \circ \tilde{\boldsymbol{\Psi}}=M_{\Delta}
$$

The relation between these maps and the various spaces and maps used in this work is summarized in the following commutative diagram. The horizontal arrows on the right are inclusion maps.

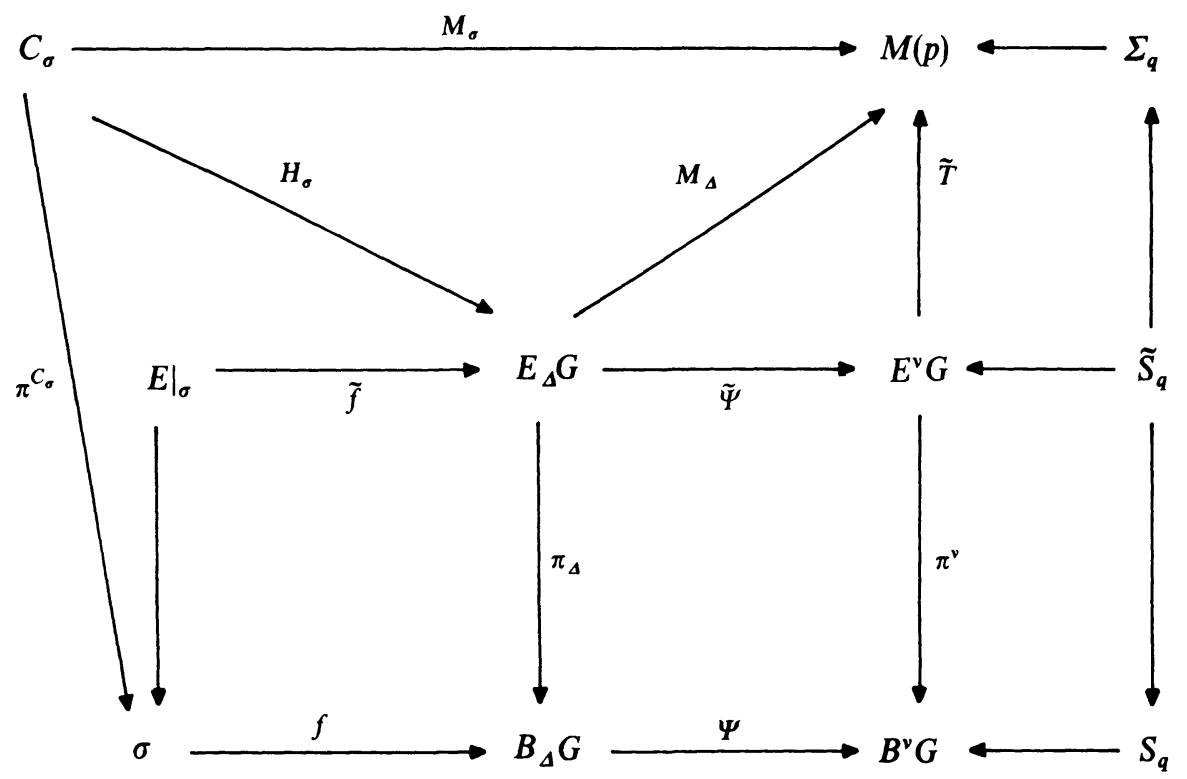

Applied to our calculation this gives

$$
\tilde{\Psi} \circ H_{\sigma}\left(C_{\sigma}\right) \cdot \tilde{S}_{q}=\tilde{T} \circ \Psi \circ H_{\sigma}\left(C_{\sigma}\right) \cdot \Sigma_{q}=M_{\sigma}\left(C_{\sigma}\right) \cdot \Sigma_{q},
$$

using $(* *)$. On the other hand, since $H_{\sigma}\left(C_{\sigma}\right)$ is in fact a section over the interior of $\sigma$, we have

$$
\tilde{\Psi} \circ H_{\sigma}\left(C_{\sigma}\right) \cdot \tilde{S}_{q}=\Psi \circ f(\sigma) \cdot S_{q}
$$

and finally

$$
C_{q}(\mathbf{V})(\sigma)=M_{\sigma}\left(C_{\sigma}\right) \cdot \Sigma_{q}
$$

The genericity hypothesis guarantees that this last intersection number is well defined; it follows that the other intersection numbers occurring in this proof, since they turn out to be equal to this one, are equally well defined.

Corollary 4.9. Let $\mathbf{u}$ be a generic $G$-valued l.g.f. on $\Lambda$, which is strictly continuous with respect to the local vertex ordering $\mathbf{0}$, and let $\xi$ be the corresponding principal $G$-bundle. Let $\mathbf{V}$ be the $G$-valued p.t.f. constructed from $\mathbf{u}$ by the method of Proposition 3.8. Then the $q^{\text {th }}$ Chern class of $\xi$ is represented by the cocycle $C_{q}(\mathbf{V})$; 
furthermore, as is clear from Paragraph 4.2, the formula for $C_{q}(\mathbf{V})$ depends only on $\mathbf{u}$.

4.10 Remark. Similar formulas could be given for the Pontrjagin classes and Euler characteristic of a generic p.t.f. with values in $G L(p, \mathbf{R})$.

\section{Computations in $U(1), S U(2)$ and $S U(3)$}

Example 5.1. $G=G L(1, \mathbf{C})$. Then $M(1)=\mathbf{C}$ and $\Sigma_{1}=\{0\}$. Let $\mathbf{u}$ be a generic $G$-valued lattice gauge field on an ordered simplicial complex $\Lambda$; assume that $\mathbf{u}$ is strictly continuous with respect to the local ordering. Let $\mathbf{V}$ be the parallel transport function constructed from $\mathbf{u}$ by the method of Proposition 3.8; let $\xi$ be the corresponding $G$-bundle; and let $C_{1}=C_{1}(\mathbf{V})$ be the cocycle representative on $\Lambda$ of the first Chern class $c_{1}(\xi) \in H^{2}(\Lambda ; \mathbf{Z})$, constructed as in Corollary 4.9. Here is what is involved in computing $C_{1}(\sigma)$, where $\sigma=\langle 012\rangle \in \Lambda$.

From paragraph 4.2 , we have

$$
M_{\sigma}\left(s_{1}, s_{2}\right)=\left(1-s_{1}\right)\left(1-s_{2}\right)+s_{1}\left(1-s_{2}\right) u_{01}+\left(1-s_{1}\right) s_{2} u_{02}+s_{1} s_{2} u_{01} u_{12} .
$$

The map $M_{\sigma}$ sends the unit square into the complex plane, and $C_{1}(\sigma)$ is the number of points sent to 0 , each counted with the sign of the Jacobian of $M_{\sigma}$ at that point with respect to the coordinates $\left(s_{1}, s_{2}\right)$ and the standard coordinates $x+i y$ in $\mathbf{C}$. It is clear that generically there are 0,1 or 2 such points, and when there are two their signs cancel.

In the special case $G=U(1) \subset G L(1, \mathbf{C})$ this procedure gives exactly the same $C_{1}(\sigma)$ that one would obtain by scaling down to $U(1)$ the $S U(2)$-algorithm of [24], i.e. constructing an as-constant-as-possible section following the local ordering and calculating the obstruction to extending that section over the 2-skeleton. This is different from the more differential-geometric method of [23], which assigned to each 2-simplex $\sigma$ a real number, the analogue of $\int_{\sigma} K d A$; the Gauss-Bonnet theorem (generalized to arbitrary circle bundles) guarantees that the two procedures give the same cohomology class.

Example 5.2. For lattice gauge fields with values in $G=S U(2)$ we could use $G=G L(2, \mathbf{C})$, but it is geometrically more natural to use $G=G L(1, \mathbf{H})$, since $S U(2)$ is also the group of unit elements in the quaternionic number system $\mathbf{H}$. (Our results extend easily to quarternionic line bundles.) Then $M(1)=\mathbf{H}$ and $\Sigma_{1}^{\prime}=\{\mathbf{0}\}$. Let $\mathbf{u}, \mathbf{V}, \xi$ be related as in the previous example, and let $C_{2}=C_{2}(\mathbf{V})$ be the cocycle representative on $\Lambda$ of $c_{2}(\xi)$, as in Corollary 4.9. Say $\sigma=\langle 01234\rangle$, oriented by the ordering. From 4.2 we have

$$
\begin{aligned}
M_{\sigma}\left(s_{1}, s_{2}, s_{3}, s_{4}\right)= & \left(1-s_{1}\right)\left(1-s_{2}\right)\left(1-s_{3}\right)\left(1-s_{4}\right) 1 \\
& +s_{1}\left(1-s_{2}\right)\left(1-s_{3}\right)\left(1-s_{4}\right) u_{01}+\cdots(\text { four terms }) \\
& +\left(1-s_{1}\right) s_{2} s_{3}\left(1-s_{4}\right) u_{02} u_{23}+\cdots(\text { six terms }) \\
& +s_{1}\left(1-s_{2}\right) s_{3} s_{4} u_{01} u_{13} u_{34}+\cdots \text { (four terms) } \\
& +s_{1} s_{2} s_{3} s_{4} u_{01} u_{12} u_{23} u_{34} .
\end{aligned}
$$


(Here 1 is the quaternionic identity element $(1,0,0,0)$.) The points where $M_{\sigma}$ meets $\Sigma_{1}^{\prime}$ are given by

$$
M_{\sigma}\left(s_{1}, s_{2}, s_{3}, s_{4}\right)=\mathbf{0}, \quad \text { with } 0 \leqq s_{i} \leqq 1 .
$$

Separating the quaternionic coordinates gives four quartic equations in four variables. The signs of the intersection points and the total intersection number are determined as in the previous example.

Again it is interesting to compare this method with that of [24] applied to the same simplex. Both methods start from the same set of sixteen points on $S^{3} \subset \mathbf{R}^{4}$. Our earlier method does a certain geodesic interpolation to obtain a map from $\partial C_{\sigma}^{4}$ to $S^{3}$, and then yields the winding number of that map. This method applies iterated linear interpolation to obtain a map from $C_{\sigma}^{4}$ to $\mathbf{R}^{4}$, and then counts how many times that map covers $\mathbf{0}$. We know that for u sufficiently continuous both algorithms must yield the same cohomology class (on the other hand it is not difficult to construct examples of strictly continuous u's for which the two methods produce different cochains). But since the geodesic method leads directly to a very simple calculation, it is preferable for this example.

If we considered $S U(2) \subset G L(2, \mathrm{C})$ instead, the map $M_{\sigma}$ would be defined just as above ( 1 is now the $2 \times 2$ identity matrix), with the interpolations taking place, nominally, in the 8-dimensional space $M(2)$. Actually since the quaternions form a linear subspace of $M(2)$ (they correspond to the matrices of the form $\left.\left(\begin{array}{cc}x+i y & z+i w \\ -z+i w & x-i y\end{array}\right)\right)$ the previous picture is just embedded in a bigger space. The locus $\Sigma_{2}$ is in this case the set of matrices with first column zero. Since this locus intersects the quaternions exactly in the origin of $\mathbf{H}$, the two calculations are in fact identical.

Example 5.3. For lattice gauge fields with values in $S U(3)$ one can generalize the geodesic method directly (see $[14,15]$ ); our method suggests using $G L(3, \mathbf{C})$. If $\Lambda$ is a 4-complex, the only relevant characteristic class is $c_{2}(\xi)$, continuing with the notation of Examples 5.1 and 5.2. The cycle $\Sigma_{2} \subset \mathbf{M}(3)=\left\{\left(A_{j}^{i}\right)\right\}$ is the set of matrices whose first two columns are linearly dependent. This set is defined by three equations, no more than two of which are independent at any point of $\Sigma_{2}$ :

$$
\left|\begin{array}{ll}
A_{1}^{1} & A_{1}^{2} \\
A_{2}^{1} & A_{2}^{2}
\end{array}\right|=0, \quad\left|\begin{array}{ll}
A_{2}^{1} & A_{2}^{2} \\
A_{3}^{1} & A_{3}^{2}
\end{array}\right|=0, \quad\left|\begin{array}{ll}
A_{1}^{1} & A_{1}^{2} \\
A_{3}^{1} & A_{3}^{2}
\end{array}\right|=0 .
$$

We abbreviate these determinants to $\varphi_{1}, \varphi_{2}, \varphi_{3}$, respectively. Since any two of the three satisfy the independence condition of paragraph 4.4 , our genericity hypothesis implies that the intersection points between $M_{\sigma}$ and $\Sigma_{2}$ will take place in the portion of $\Sigma_{2}$ where any two of the three equations are independent.

Let $C_{2}=C_{2}(\mathbf{V})$ be the cocycle representative of $c_{2}(\xi)$, as in Corollary 4.9. Say $\sigma=\langle 01234\rangle$, with vertices so ordered. The function $M_{\sigma}: C_{\sigma}^{4} \rightarrow \mathbf{M}(3)$ is given by the same formula as in Example 5.2 (except now 1 is the $3 \times 3$ identity matrix). Substituting this expression into the real and imaginary parts of the equations $\varphi_{2}=0, \varphi_{3}=0$ leads to four equations of degree 8 in the four unknowns $s_{1}, s_{2}, s_{3}$ 
and $s_{4}$ :

$$
(*)\left\{\begin{array}{l}
\mathfrak{R} \varphi_{2}\left(M_{\sigma}\left(s_{1}, s_{2}, s_{3}, s_{4}\right)\right)=0 \\
\mathfrak{I} \varphi_{2}\left(M_{\sigma}\left(s_{1}, s_{2}, s_{3}, s_{4}\right)\right)=0 \\
\mathfrak{R} \varphi_{3}\left(M_{\sigma}\left(s_{1}, s_{2}, s_{3}, s_{4}\right)\right)=0 \\
\mathfrak{I} \varphi_{3}\left(M_{\sigma}\left(s_{1}, s_{2}, s_{3}, s_{4}\right)\right)=0 \\
s_{i} \text { real, } 0 \leqq s_{i} \leqq 1 .
\end{array}\right.
$$

Each solution of these equations and inequalities will be an intersection point $p$ of $M_{\sigma}$ and $\Sigma_{2}$. Before discussing methods for solving $(*)$, let us check the sign of $p$. Let $\gamma_{2}=\nabla \varphi_{2}(p)$ as in [20] and $\gamma_{3}=\nabla \varphi_{3}(p)$. They define a complex 2-dimensional subspace $L$ of $\mathbf{M}(3)$, with a canonical orientation given by $\left(\mathfrak{R} \gamma_{2}, \mathfrak{I} \gamma_{2}, \mathfrak{R} \gamma_{3}, \mathfrak{I} \gamma_{3}\right)$. By the genericity hypothesis, the projections into $L$ of the vectors $\left(\partial / \partial s_{i}\right) M_{\sigma}(p)$, $i=1,2,3,4$, form a real basis of $L$. The sign of $p$ is + or - according as this basis agrees or disagrees in orientation with the canonical one.

Finally

$$
C_{2}(\sigma)=\sum_{\text {intersection points } p} \pm 1
$$

plus or minus following the sign of $p$.

Detailed consideration of the methods for solving a system like $(*)$ is beyond the scope of this work. There are two main types of scheme. One, explained in [5], uses elimination theory to reduce $(*)$ to a system which can be solved by repeating extraction of roots of polynomials of one variable. The other (see [16] and the references therein) can be called the homotopy method: one constructs a homotopy between $(*)$ and a similar system $(* *)$ all of whose roots are known. Then one can follow the homotopy backwards, tracking the roots of $(* *)$; generically they will lead to those of $(*)$.

Whichever method one uses to solve $(*)$, using intersection numbers expresses the topological charge as a sum of integers. It therefore avoids the round-off errors associated with a computation shceme expressing the topological charge as a curvature integral and using numerical integration, errors which can only be aggravated by increased lattice size.

On the other hand both the intersection scheme and the integration scheme involve a fixed amount of computation per simplex, whereas in a scheme involving cooling the entire lattice the computation time is sure to grow much more rapidly as a function of the total number of simplexes.

\section{Proofs of Propositions on Equivalence and Generic Approximation}

This section contains the proofs of Propositions 3.10 and 4.7.

Proof of Proposition 3.10. We shall construct a family

$$
\mathbf{W}=\left\{W_{\sigma}: \sigma \in \Lambda, \operatorname{dim} \sigma \geqq 1\right\}
$$

of maps $W_{\sigma}: c_{\sigma}^{r-1} \times[0,1] \rightarrow M(p)$ such that, for each $\sigma$, 
(a) writing $\sigma$ as $\langle 0, \ldots, r\rangle$, we have

$$
\left\|W_{\sigma}\left(s_{1}, \ldots, s_{r-1}, t\right)-u_{0 r}\right\|<2(2 r-1) K^{r} \delta .
$$

(Recall that strict continuity means $\delta<1 / 4 K^{2} n \varphi(K, n)$, and therefore $2(2 r-1) K^{r} \delta<$ $4 n K^{r} \delta<1 / K$ for $0 \leqq r \leqq n$; so condition (a) implies that $W_{\sigma}$ takes values in $G$.)

(b) for each $t$, set $V_{\sigma}^{t}=\left.W_{\sigma}\right|_{c_{\sigma}^{r-1} \times\{t\}}$; then $\left\{V_{\sigma}^{t}\right\}$ is a (G-valued) p.t.f. $\mathbf{V}^{t}$ on $\Lambda$.

(c) $V_{\sigma}^{0}=V_{\sigma} ; V_{\sigma}^{1}=V_{\sigma}^{\prime}$;

Once $\mathbf{W}$ has been constructed, the proposition quickly follows; for if $\xi^{t}$ is the bundle over $|\Lambda|$ determined by $\mathbf{V}^{t}$, then $\left\{\xi^{t}\right\}$ is a one-parameter family of bundles joining $\xi^{0}=\xi$ to $\xi^{1}=\xi^{\prime}$; so $\xi$ and $\xi^{\prime}$ are isomorphic.

We construct $W_{\sigma}$ by induction on $r=\operatorname{dim} \sigma$. When $r=1, W_{\sigma}: I \rightarrow M(p)$ is determined on $\partial I$ by condition (c): $W_{\sigma}(0)=v_{\langle 01\rangle}, W_{\sigma}(1)=v_{\langle 01\rangle}^{\prime}$. By hypothesis, $\left\|v_{\langle 01\rangle}-u_{01}\right\|<\delta$, and the same holds for $v_{\langle 01\rangle}^{\prime}$. That is, $W_{\sigma}(\partial I) \subseteq B\left(u_{01}, \delta\right)$. Let $W_{\sigma}: I \rightarrow B\left(u_{01}, \delta\right)$ be any extension of $\left.W_{\sigma}\right|_{\partial I}$. Since $\delta<2 K \delta$, condition (a) holds, while condition (b) is automatic in dimension 1.

Now assume $W_{\tau}$ has been constructed whenever $\operatorname{dim} \tau\langle r$, and say $\sigma=\langle 0, \ldots, r\rangle$. Then $W_{\sigma}$ is prescribed on $\partial\left(c_{\sigma}^{r-1} \times I\right)$ by (b) and (c), as follows:

(i) On $c_{\sigma}^{r-1} \times \partial I, W_{\sigma}$ is given by condition (c).

(ii) On $\left(\partial_{j} c_{\sigma}^{r-1}\right) \times I$, for $j=1, \ldots, r-1$, condition (b) forces $W_{\sigma}\left(s_{1}, \ldots, s_{j}=0, \ldots, t\right)=$ $W_{\partial_{j} \sigma}\left(s_{1}, \ldots, \hat{s}_{j}, \ldots, t\right)$.

(iii) On $\left(\partial^{j} c_{\sigma}^{r-1}\right) \times I$, for $j=1, \ldots, r-1$, condition (b) forces $W_{\sigma}\left(s_{1}, \ldots, s_{j}=1, \ldots, t\right)=$ $W_{\langle 0, \ldots, j\rangle}\left(s_{1}, \ldots, s_{j-1}, t\right) \cdot W_{\langle j, \ldots, r\rangle}\left(s_{j+1}, \ldots, s_{r-1}, t\right)$. (We omit the verification that these prescriptions are compatible.) We now claim that $W_{\sigma}$ satisfies (a) on $\partial\left(c_{\sigma}^{r-1} \times I\right)$. This follows from Proposition 3.8 (2) in case (i) since $\varphi(K, r) \delta \leqq r K^{r-1} \delta \leqq$ $2(2 r-1) K^{r} \delta$, and from the inductive hypothesis in case (ii). For case (iii), set $\sigma^{\prime}=\langle 0, \ldots, j\rangle, \sigma^{\prime \prime}=\langle j, \ldots, r\rangle, \vec{s}=\left(s_{1}, \ldots, s_{r-1}\right), \vec{s}^{\prime}=\left(s_{1}, \ldots, s_{j-1}\right), \vec{s}^{\prime \prime}=\left(s_{j+1}, \ldots, s_{r-1}\right)$. Then

$$
\begin{aligned}
W_{\sigma}(\vec{s}, t)-u_{0 r}= & \left(W_{\sigma^{\prime}}\left(\vec{s}^{\prime}, t\right)-u_{0 j}\right) \cdot\left(W_{\sigma^{\prime \prime}}\left(\vec{s}^{\prime \prime}, t\right)-u_{j r}\right)+u_{0 j} \cdot\left(W_{\sigma^{\prime \prime}}\left(\vec{s}^{\prime \prime}, t\right)-u_{j r}\right) \\
& +\left(W_{\sigma^{\prime}}\left(\vec{s}^{\prime}, t\right)-u_{0 j}\right) \cdot u_{j r}+u_{0 j} \cdot u_{j r}-u_{0 r} .
\end{aligned}
$$

Applying condition (a) to $W_{\sigma^{\prime}}$ and $W_{\sigma^{\prime \prime}}$ and using the fact that each $\left\|u_{i j}\right\| \leqq K$, gives

$$
\begin{aligned}
\left\|W_{\sigma}(\vec{s}, t)-u_{0 r}\right\| \leqq & 4(2 j-1)(2(r-j)-1) \delta^{2} K^{r} \\
& +K \cdot 2(2(r-j)-1) K^{r-j} \delta \\
& +2(2 j-1) K^{j} \delta \cdot K \\
& +\delta .
\end{aligned}
$$

Now $(2 j-1)(2(r-j)-1)<4 j(n-j) \leqq n^{2}$, so the first term is dominated by $4 n^{2} \delta^{2} K^{r}$ which in turn, using strict continuity, is dominated by $\delta K^{r}$; also here $1 \leqq j \leqq r-1$ so $K^{r-j+1}$ and $K^{j+1}$ are both $\leqq K^{r}$, and the sum of the two middle terms is dominated by $2(2 r-2) K^{r} \delta$; hence

$$
\begin{aligned}
\left\|W_{\sigma}(\vec{s}, t)-u_{0 r}\right\| & <\delta K^{r}+2(2 r-2) \delta K^{r}+\delta K^{r} \\
& <2(2 r-1) K^{r} \delta .
\end{aligned}
$$

Thus $W_{\sigma}$ satisfies condition (a) on $\partial\left(c_{\sigma}^{r-1} \times I\right)$. 
Define $W_{\sigma}: c_{\sigma}^{r-1} \times I \rightarrow B\left(u_{0 r}, 2(2 r-1) K^{r} \delta\right)$ to be any extension of $\left.W_{\sigma}\right|_{\partial\left(c_{\sigma}^{r-1} \times I\right)}$. This completes the inductive step in the construction of the $W_{\sigma}$, and the proof of the proposition.

Proof of Proposition 4.7. The existence of an open neighborhood $U$ consisting of strictly continuous l.g.f.'s $\mathbf{u}$ which determine the same bundle as $\mathbf{u}^{0}$ is given by Corollary 3.14. It remains to show that the generic u's form an open, dense subset $U^{\prime} \subset U$.

Let $\mathbf{u}$ be an element of $U$; let $\mathbf{V}$ be the corresponding p.t.f., constructed according to Proposition 3.8; for each $\sigma=\langle 0, \ldots, r\rangle$, let $H_{\sigma}: C_{\sigma}^{r} \rightarrow E_{\Delta} G$ be constructed from $\mathbf{V}$ as in Definition 2.3; set $M_{\sigma}^{\mathbf{u}}=M_{\Delta}{ }^{\circ} H_{\sigma}$, and for $\vec{s} \in C_{\sigma}^{r}$, set $F_{\sigma}^{\vec{s}}(\mathbf{u})=M_{\sigma}^{\mathbf{u}}(\vec{s})$. This gives a map $F_{\sigma}^{\vec{s}}: U \rightarrow M(p)$. (In fact, $F_{\sigma}^{\vec{s}}$ only depends on $\left\{u_{i j}:\langle i j\rangle\langle\sigma\}\right.$.) The function $F_{\sigma}^{\vec{s}}$ is clearly smooth. Note that $F_{\sigma}^{\overrightarrow{0}}(\mathbf{u})=1$ for every $\mathbf{u}$. We shall now show that, provided $\vec{s} \neq \overrightarrow{0}, F_{\sigma}^{\vec{s}}$ has maximal rank at every $\mathbf{u} \in U$; that is, that the map of tangent spaces:

$$
\text { (*) } \quad T_{\mathbf{u}} F_{\sigma}^{\vec{s}}: T_{\mathbf{u}}\left(G^{z}\right) \rightarrow T_{F_{\sigma}^{s}(\mathbf{u})} M(p)
$$

is surjective; here $z$ is the number of 1 -simplexes of $\Lambda$.

We start from the formula of paragraph 4.2:

$$
(* *) \quad F_{\sigma}^{\vec{s}}(\mathbf{u})=\sum_{J} \lambda_{J}(\vec{s}) \cdot \tilde{u}_{J} .
$$

We shall prove $(*)$ by induction on the number of vertices $i$ along $1, \ldots, r$ such that $s_{i}$ is equal to either 0 or 1 (but without restriction on $r=\operatorname{dim} \sigma$ ).

Case 1. None of the $s_{i}$ is 0 or 1 . In this case, the dependence of $F_{\sigma}^{\vec{s}}$ on $u_{0 r}$ in $(* *)$ occurs only in the term $\lambda_{\{r\}}(\vec{s}) u_{0 r}$, with $J=\{r\}$. Here $\lambda_{\{r\}}(\vec{s})=s_{r} \prod_{j=1}^{r-1}\left(1-s_{j}\right) \neq 0$.
Hence the matrix

$$
\frac{\partial}{\partial u_{0 r}} F_{\sigma}^{\vec{s}}(\mathbf{u})
$$

of partial derivatives of $F_{\sigma}^{\vec{s}}$ with respect to the entries of $u_{0 r}$ is equal to $\lambda_{\{r\}}(\vec{s})$ times a $p^{2} \times p^{2}$ identity matrix. So $(*)$ clearly holds in this case.

Case 2. Some $s_{j}=0$. Set $\rho=\partial_{j} \sigma$, and $\vec{s}^{\prime}=\left(s_{1}, \ldots, \hat{s}_{j}, \ldots, s_{r}\right)$. Then $F_{\sigma}^{\vec{s}}=F_{\sigma}^{\vec{s} \prime}$, and in $\vec{s}^{\prime}$ there is one fewer index that has the value 0 or 1 . So $(*)$ holds by the induction hypothesis.

Case 3. No $s_{j}=0$, but some $s_{j}=1$. Let $j$ denote the largest index such that $s_{j}=1$. Set $\tau^{\prime}=\langle 0, \ldots, j\rangle, \tau^{\prime \prime}=\langle j, \ldots, r\rangle, \vec{s}^{\prime}=\left(s_{1}, \ldots, s_{j}=1\right), \vec{s}^{\prime \prime}=\left(s_{j+1}, \ldots, s_{r}\right)$. Then

$$
F_{\sigma}^{\vec{s}}(\mathbf{u})=F_{\tau^{\prime}}^{\vec{s}^{\prime}}(\mathbf{u}) \cdot F_{\tau^{\prime}}^{\vec{s}^{\prime \prime}}(\mathbf{u}) \text {. }
$$

Now $F_{\tau^{\prime}}$ and $F_{\tau^{\prime \prime}}$ depend only on $\left\{u_{i j}:\langle i j\rangle\left\langle\tau^{\prime}\right\}\right.$ and $\left\{u_{i j}:\langle i j\rangle\left\langle\tau^{\prime \prime}\right\}\right.$, respectively, and these are disjoint sets of variables. Since none of $s_{j+1}, \ldots, s_{r}$ is 0 or 1 , we know by Case 1 that $\partial / \partial u_{j+1, r} F_{\tau^{\prime \prime}}^{\overrightarrow{s^{\prime \prime}}}$ is nonsingular at $\mathbf{u}$. Also, since $F_{\tau^{\prime}}^{\vec{s}^{\prime}}(\mathbf{u})=V_{\tau^{\prime}}^{\mathbf{u}}\left(s_{1}, \ldots, s_{j-1}\right)$ is a non-singular $p \times p$ matrix, left-multiplication by $F_{\tau^{\prime}}^{\vec{s}^{\prime}}(\mathbf{u})$ is a diffeomorphism $M(p) \rightarrow M(p)$; it follows from the chain rule that $\partial / \partial u_{j+1, r} F_{\sigma}^{\vec{s}}$ is nonsingular at $\mathbf{u}$, so $(*)$ holds in this case too. 
For each $\sigma$, we now apply [1, Theorem 19.1] to the map $U \rightarrow \mathscr{C}^{\infty}\left(C_{\sigma}^{r}, M(p)\right)$ given by $\mathbf{u} \mapsto M_{\sigma}^{\mathbf{u}}$. We conclude that the subset $U_{\sigma}$ of $U$, consisting of these $\mathbf{u}$ such that $M_{\sigma}^{\mathrm{u}}$ is transverse to $\Sigma_{q} \subset M(p)$, is open and dense in $U$. Then $U^{\prime}=\bigcap\left\{U_{\sigma}: \sigma \in \Lambda\right\}$ is the required open, dense subset of $U$ consisting of generic l.g.f's.

\section{Appendix. Parallel Transport Functions and Coordinate Bundles}

Our purpose in this appendix is to demonstrate, for a fixed simplicial complex $\Lambda$ with local vertex ordering $\mathbf{0}$, an algorithm that assigns to each parallel transport function $\mathbf{V}$ on $\Lambda$ a coordinate bundle $\mathbf{v}$ on $\Lambda$ of the type constructed in [24] and another algorithm in the reverse direction, such that in each case the corresponding $G$-bundles are isomorphic.

A.1 Notation. Coordinate bundles will have slightly different notation from that of [24].

First we summarize from [24]: Let $\sigma=\langle 0, \ldots, r\rangle$ be a simplex of $\Lambda$ (vertices written in increasing o-order). The dual cell block $c_{i}^{\sigma}, i=0, \ldots, r$ is defined in terms of the barycentric coordinates $\left(t_{0}, \ldots, t_{r}\right)$ by

$$
c_{i}^{\sigma}=\left\{\left(t_{0}, \ldots, t_{r}\right) \mid t_{i} \geqq \text { all } t_{j}\right\} .
$$

Set $c_{i j}^{\sigma}=c_{i}^{\sigma} \cap c_{j}^{\sigma}$, etc; $c_{\tau}^{\sigma}=\bigcap_{i \in \tau} c_{i}^{\sigma}$, for $\tau \prec \sigma$; and let the $d u a l$ cell dual to vertex $i$ be

$$
c_{i}=\bigcup_{\sigma \ni l} c_{i}^{\sigma} .
$$

A.2. A coordinate bundle $\mathbf{v}$ on $\Lambda$ consists of a family of maps $\left\{v_{i j}: c_{i j} \rightarrow G\right\}_{\langle i j\rangle \in \Lambda}$ such that

$$
\begin{array}{lll}
v_{j i}(x)=v_{i j}(x)^{-1}, & \text { for } & x \in c_{i j}, \\
v_{i k}(x)=v_{i j}(x)^{\prime} \cdot v_{j k}(x), & \text { for } & x \in c_{i j k} .
\end{array}
$$

From $\mathbf{v}$ we construct a principal $G$-bundle $\xi$ over $X$ from the disjoint union $\cup \cup c_{i} \times G$ by identifying $\left(x, g_{i}\right) \in c_{i} \times G$ with $\left(x, v_{i j}(x) \cdot g_{j}\right) \in c_{j} \times G$, for $x \in c_{i j}$.

A.3. In [24] we worked with certain coordinate bundles that we shall now call o-adapted. First, we observed that it is convenient to replace barycentric coordinates on $c_{i}^{\sigma}$ by the modified barycentric coordinates $\left(s_{0}^{\prime}, \ldots, \hat{s}_{i}^{\prime}, \ldots, s_{r}^{\prime}\right)$ given by $s_{j}^{\prime}=t_{j} / t_{i}$. In these coordinates $c_{i}^{\sigma}$ is the cube $\left\{s_{i}^{\prime}=1,0 \leqq s_{j}^{\prime} \leqq 1\right.$ for $\left.j \neq i\right\}$, while $c_{i j}=\left\{s_{i}^{\prime}=s_{j}^{\prime}=1,0 \leqq s_{k}^{\prime} \leqq 1\right.$ for $\left.k \neq i, j\right\}$, etc.

Definition A.4. A coordinate bundle $\mathbf{v}$ is $\mathbf{0}$-adapted if on each $c_{i j}$ (here we assume $i<j$ ) the transition function $v_{i j}$ is a function only of $\left\{s_{k}^{\prime} \mid i<k<j\right\}$.

$A .5$. It follows from [24] that, given any principal $G$-bundle $\xi$ over $X$, if $\Lambda$ is a sufficiently fine triangulation of $X$, then $\xi$ can be represented by an o-adapted coordinate bundle on $\Lambda$.

A.6. For our present purposes we find it more convenient to use variants of these constructions. Recall the standard projection $\pi^{C^{r}}: C^{r} \rightarrow \sigma^{r}$ from Definition 1.3; it is given in terms of coordinates $s_{1}, \ldots, s_{r}, 0 \leqq s_{i} \leqq 1$ on $C^{r}$ and barycentric coordinates 
$\left(t_{0}, \ldots, t_{r}\right)$ on $\sigma^{r}$ by

$$
\begin{array}{cr}
t_{0}=\left(1-s_{1}\right)\left(1-s_{2}\right) \cdots\left(1-s_{r}\right) \\
t_{1}= & s_{1}\left(1-s_{2}\right) \cdots\left(1-s_{r}\right) \\
\vdots & \\
t_{r}= & s_{r} .
\end{array}
$$

The inverse relations, when defined, are

$$
\begin{aligned}
& s_{1}=t_{1} /\left(1-\sum_{j=2}^{r} t_{j}\right) \\
& s_{2}=t_{2} /\left(1-\sum_{j=3}^{r} t_{j}\right) \\
& \vdots \\
& s_{r}=t_{r} .
\end{aligned}
$$

We shall use the $s_{i}$ 's as (over-determined) coordinates on $\sigma^{r}$.

Definition A.7. The quasi-dual cell blocks $\tilde{c}_{i}^{\sigma}, i=1, \ldots, r$ are defined by $\tilde{c}_{i}^{\sigma}=$ $\left\{\left(s_{1}, \ldots, s_{r}\right) \mid s_{i} \geqq \frac{1}{2}\right.$ and $s_{j} \leqq \frac{1}{2}$ for $\left.j>i\right\}$ if $i \geqq 1$, and $\tilde{c}_{0}^{\sigma}=\left\{\left(s_{1}, \ldots, s_{r}\right) \mid\right.$ all $\left.s_{j} \leqq \frac{1}{2}\right\}$. (See Fig. 3.)

Set $\tilde{c}_{i j}^{\sigma}=\tilde{c}_{i}^{\sigma} \cap \tilde{c}_{j}^{\sigma}$, etc., for $\tau \prec \sigma$ set $\tilde{c}_{\tau}^{\sigma}=\bigcap_{i \in \tau} \tilde{c}_{i}^{\sigma}$ and define the quasi-dual cell $\tilde{c}_{i}=\bigcup \tilde{c}_{\sigma \ni i}^{\sigma}$.

Lemma A.8. The complex $\left\{\tilde{c}_{\tau}^{\sigma} \mid \tau \prec \sigma\right.$ in $\left.\Lambda\right\}$ is combinatorially isomorphic to the complex $\left\{c_{\tau}^{\sigma}\right\}$, in such a way that each simplex of $\Lambda$ is preserved.

Proof. We will construct $\left\{\tilde{c}_{\tau}^{\sigma}\right\}$ as an amalgamation of a "quasi-barycentric" subdivision $\tilde{\Lambda}^{(1)}$ of $\Lambda$. Let $\hat{\sigma}$ denote the barycenter of $\sigma$; then

$$
c_{\tau}^{\sigma}=\bigcup_{\tau \prec \tau_{0} \prec \cdots \prec \tau_{s} \prec \sigma}\left\langle\hat{\tau}_{0}, \ldots, \hat{\tau}_{s}\right\rangle .
$$

Now for each $\sigma=\langle 0, \ldots, r\rangle$, define its quasi-barycenter $\tilde{\sigma}$ to be the point with coordinates

$$
s_{i}(\tilde{\sigma})=1 / 2^{r-i+1} \quad \text { for } \quad i=1, \ldots, r
$$

or

$$
t_{0}(\tilde{\sigma})=1 / 2^{r}, t_{i}(\tilde{\sigma})=1 / 2^{r-i+1} \quad \text { for } \quad i \geqq 1
$$

With these points as new vertices, we can construct a quasi-barycentric subdivision $\tilde{\Lambda}^{(1)}$ of $\Lambda$ (see Fig. 3); and clearly $\tilde{\Lambda}^{(1)}$ is simplicially isomorphic to the standard first barycentric subdivision $\Lambda^{(1)}$. Now

$$
\tilde{c}_{\tau}^{\sigma}=\bigcup_{\tau \prec \tau_{0} \prec \cdots \prec \tau_{s} \prec \sigma}\left\langle\tilde{\tau}_{0}, \ldots, \tilde{\tau}_{s}\right\rangle .
$$

So the isomorphism $\tilde{\Lambda}^{(1)} \approx \Lambda^{(1)}$ induces an isomorphism $\left\{\tilde{c}_{\tau}^{\sigma}\right\} \approx\left\{c_{\tau}^{\sigma}\right\}$. 
(b)
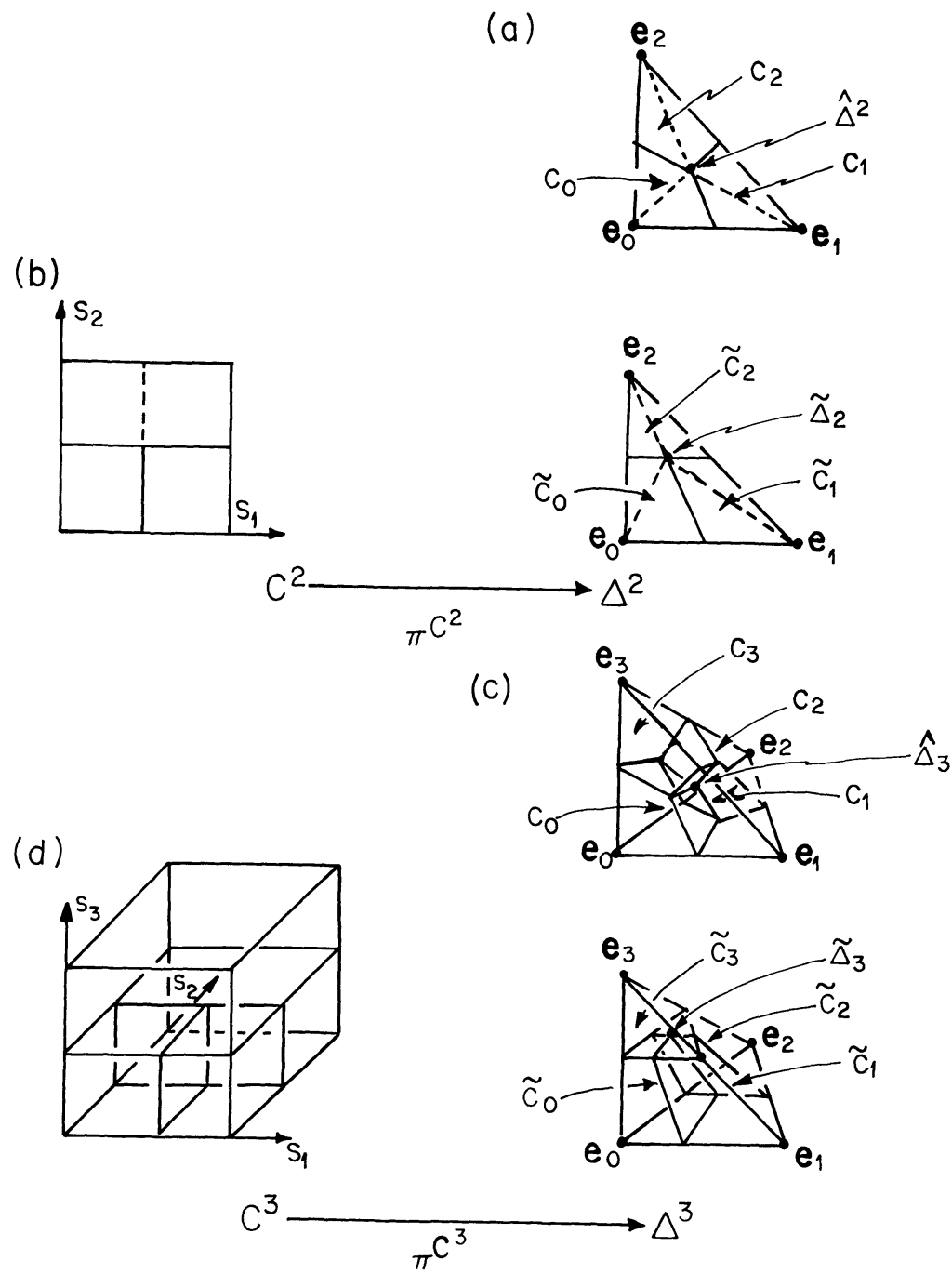

Fig. 3. (a) dual cell blocks in $\Delta^{2}$; (b) quasi-dual cell blocks in $\Delta^{2}$; (c) dual cell blocks and (d) quasi-dual cell blocks in $\Delta^{3}$

A.9. It follows that we can use the quasi-dual cells $\left\{\tilde{c}_{i}\right\}$ as domains of charts for any bundle over $X$, and the transition functions of a coordinate bundle $\tilde{\mathbf{v}}$ will now be maps

$$
\tilde{v}_{i j}: \tilde{c}_{i j} \rightarrow G,
$$

satisfying conditions analogous to the previous ones.

On $\tilde{c}_{i j k}^{\sigma}=\left\{\left(s_{1}, \ldots, s_{r}\right) \mid s_{i} \geqq \frac{1}{2}, s_{l} \leqq \frac{1}{2}\right.$ for $l>i$, and $\left.s_{j}=s_{k}=\frac{1}{2}\right\}$ the cocycle condition becomes (for $i<j<k$ )

$$
\tilde{v}_{i k}\left(s_{1}, \ldots, s_{j}=\frac{1}{2}, \ldots, s_{r}\right)=\tilde{v}_{i j}\left(s_{1}, \ldots, s_{k}=\frac{1}{2}, \ldots, s_{r}\right) \tilde{v}_{j k}\left(s_{1}, \ldots, s_{j}=\frac{1}{2}, \ldots, s_{r}\right) .
$$


As before, we say that $\tilde{\mathbf{v}}$ is $\mathbf{0}$-adapted if, on each $\tilde{c}_{i j}, \tilde{v}_{i j}$ is only a function of $\left\{s_{k} \mid i<k<j\right\}$.

A.10. Define $\mu: I \rightarrow\left[0, \frac{1}{2}\right]$ by

$$
\mu(s)= \begin{cases}s, & 0 \leqq s \leqq \frac{1}{2} \\ \frac{1}{2}, & \frac{1}{2} \leqq s \leqq 1\end{cases}
$$

For each $t \in\left[\frac{1}{2}, 1\right]$, define $\lambda_{t}: I \rightarrow I$ by

$$
\lambda_{t}(s)= \begin{cases}s / t, & 0 \leqq s \leqq t \\ 1, & s \geqq t\end{cases}
$$

and set $\lambda=\lambda_{1 / 2}$, so $\mu(s)=\lambda(s) / 2$.

A.11. Now, given an $\mathbf{0}$-adapted coordinate bundle $\tilde{\mathbf{v}}$, define $V_{\sigma}: C^{r-1} \rightarrow G$, where $\sigma=\langle 0, \ldots, r\rangle$, by

$$
V_{\sigma}\left(s_{1}, \ldots, s_{r-1}\right)=\tilde{v}_{0 r}^{\sigma}\left(\mu\left(s_{1}\right), \ldots, \mu\left(s_{r-1}\right)\right),
$$

and set $\mathbf{V}(\tilde{\mathbf{v}})=\left\{V_{\sigma} \mid \operatorname{dim} \sigma \geqq 1\right\}$. Conversely, given a p.t.f. $\mathbf{V}$ on $\Lambda$, define $\tilde{v}_{i j}^{\sigma}: \tilde{c}_{i j}^{\sigma} \rightarrow G$ by

$$
\tilde{v}_{i j}^{\sigma}\left(s_{1}, \ldots, s_{j}=\frac{1}{2}, \ldots, s_{r}\right)=V_{\langle i, i+1, \ldots, j\rangle}\left(\lambda\left(s_{i+1}\right), \ldots, \lambda\left(s_{j-1}\right)\right) .
$$

For fixed $i$ and $j$, the $\tilde{v}_{i j}^{\sigma}$ define a map $\tilde{v}_{i j}: \tilde{c}_{i j} \rightarrow G$; let $\tilde{\mathbf{v}}(\mathbf{V})=\left\{\tilde{v}_{i j}\right\}_{\langle i j\rangle \in \Lambda}$.

\section{Proposition A.12}

1. $\mathbf{V}(\tilde{\mathbf{v}})$ is $a$ p.t.f. on $\Lambda$.

2. $\tilde{\mathbf{v}}(\mathbf{V})$ is an $\mathbf{0}$-adapted coordinate bundle over $\Lambda$.

3. In either case, the bundles $\xi$ constructed from $\mathbf{V}$ and $\tilde{\xi}$ from $\tilde{\mathbf{v}}$ are isomorphic.

Proof. The proofs of (1) and (2) are immediate. Now let $\tilde{\mathbf{v}}$ be given, and set $\mathbf{V}=\mathbf{V}(\tilde{\mathbf{v}})$. We shall display charts for $\xi$ for which the transition functions are precisely the $\tilde{v}_{i j}$ 's.

To simplify the exposition in this proof, we shall assume the vertices of $\Lambda$ are totally ordered.

Let $\pi_{\Delta}: E_{\Delta} G \rightarrow B_{\Delta} G$ be Milnor's universal bundle, and let $f: X \rightarrow B_{\Delta} G$ be the classifying map for $\xi$ given in Proposition 2.4. Consider $\sigma=\langle 0, \ldots, r\rangle \in \Lambda$ and set $\sigma_{i}=\langle 0, \ldots, i\rangle$ for $0 \leqq i \leqq r$. Then $f$ is defined on $\sigma$ by

$$
f\left(t_{0}, \ldots, t_{r}\right)=\pi_{\Delta}\left(t_{0} \mathbf{e}_{0}+t_{1}\left(V_{\sigma_{1}}\right) \cdot \mathbf{e}_{1}+t_{2}\left(V_{\sigma_{2}}\left(s_{1}\right)\right) \cdot \mathbf{e}_{2}+\cdots+t_{r}\left(V_{\sigma}\left(s_{1}, \ldots, s_{r-1}\right)\right) \cdot \mathbf{e}_{r}\right) .
$$

As open charts for the universal bundle, we may take the

$$
U_{i}=\left\{\pi_{\Delta}\left(\sum t_{j} g_{j} \cdot \mathbf{e}_{j}\right) \mid t_{i}>0\right\} .
$$

Then $\left.E_{\Delta} G\right|_{U_{i}}=\left\{\sum t_{j} g_{j} \cdot \mathbf{e}_{j} \mid t_{i}>0\right\}$, and the fibre coordinate at $\sum t_{j} g_{j} \cdot \mathbf{e}_{j}$ is $g_{i}$. Thus the transition function $u_{i j}: U_{i} \cap U_{j} \rightarrow G$ is given by

$$
u_{i j}\left(\pi_{\Delta}\left(\sum t_{j} g_{j} \cdot \mathbf{e}_{j}\right)\right)=g_{i}^{-1} g_{j}
$$

Now $f\left(\tilde{c}_{i}\right) \subseteq U_{i}$, so we may use the induced charts on $\tilde{c}_{i}$, yielding $\left\{v_{i j}=\right.$ $\left.u_{i j} \circ f: \tilde{c}_{i j} \rightarrow G\right\}$ as transition functions for $\xi$. Thus, when $s_{i} \geqq \frac{1}{2}, s_{k} \leqq \frac{1}{2}$ for $k>i$, and 
$s_{j}=\frac{1}{2}$ we have

$$
\begin{aligned}
v_{i j}^{\sigma}\left(s_{1}, \ldots, s_{r-1}\right) & =V_{\sigma_{l}}\left(s_{1}, \ldots, s_{i-1}\right)^{-1} \cdot V_{\sigma_{j}}\left(s_{1}, \ldots, s_{j-1}\right) \\
& =\tilde{v}_{0 i}^{\sigma}\left(\mu\left(s_{1}\right), \ldots, \mu\left(s_{i-1}\right)\right)^{-1} \cdot \tilde{v}_{0 j}^{\sigma}\left(\mu\left(s_{1}\right), \ldots, \mu\left(s_{i-1}\right), 1_{i}, s_{i+1}, \ldots, s_{j-1}\right) \\
& =\tilde{v}_{0 i}^{\sigma}\left(\mu\left(s_{1}\right), \ldots, \mu\left(s_{i-1}\right)\right)^{-1} \cdot\left[\tilde{v}_{0 i}^{\sigma}\left(\mu\left(s_{1}\right), \ldots, \mu\left(s_{i-1}\right) \cdot \tilde{v}_{i j}^{\sigma}\left(s_{i+1}, \ldots, s_{j-1}\right)\right]\right. \\
& =\tilde{v}_{i j}^{\sigma}\left(s_{i+1}, \ldots, s_{j-1}\right) \\
& =\tilde{v}_{i j}^{\sigma}\left(s_{1}, \ldots, s_{r-1}\right), \quad \text { since } \tilde{\mathbf{v}} \text { is o-adapted. }
\end{aligned}
$$

Thus the $\tilde{v}_{i j}$ are the transition functions for $\xi$.

Now let $\mathbf{V}$ be given, and set $\tilde{\mathbf{v}}=\tilde{\mathbf{v}}(\mathbf{V})$. We shall show that the $\tilde{v}_{i j}$ 's are transition functions for a bundle $\xi^{*}$ isomorphic to $\xi$. For each $t \in\left[\frac{1}{2}, 1\right]$, define $V_{\sigma}^{t}: C^{r-1} \rightarrow G$ by

$$
V_{\sigma}^{t}\left(s_{1}, \ldots, s_{r-1}\right)=V_{\sigma}\left(\lambda_{t}\left(s_{1}\right), \ldots, \lambda_{t}\left(s_{r-1}\right)\right) .
$$

Then $\mathbf{V}^{t}=\left\{V_{\sigma}^{t} \mid \operatorname{dim} \sigma \geqq 1\right\}$ is a p.t.f. on $\Lambda$, and therefore induces a bundle $\xi^{t}$ over $X$. So $\xi^{1}=\xi$ is isomorphic to $\xi^{1 / 2}$. To complete the proof, taking $\xi^{*}=\xi^{1 / 2}$, it is enough to show that $\mathbf{V}(\tilde{\mathbf{v}})=\mathbf{V}^{1 / 2}$. Unraveling the definitions we obtain

$$
\begin{aligned}
(V(\tilde{\mathbf{v}}))_{\sigma}\left(s_{1}, \ldots, s_{r-1}\right) & =\tilde{v}_{0 r}^{\sigma}\left(\mu\left(s_{1}\right), \ldots, \mu\left(s_{r-1}\right)\right) \\
& =V_{\sigma}\left(\lambda\left(\mu\left(s_{1}\right)\right), \ldots, \lambda\left(\mu\left(s_{r-1}\right)\right)\right) \\
& =V_{\sigma}\left(\lambda\left(s_{1}\right), \ldots, \lambda\left(s_{r-1}\right)\right) \\
& =V_{\sigma}^{1 / 2}\left(s_{1}, \ldots, s_{r-1}\right) .
\end{aligned}
$$

Acknowledgements. We thank Charles Patton for a useful conversation, and the first referee for a helpful critique of an earlier version of this paper.

\section{References}

1. Abraham, R., Robbin, J.: Transversal Mappings and Flows. New York: Benjamin 1967

2. Adams, J.: On the cobar construction. In: Centre Belge de Recherches Mathématiques, Colloque de Topologie Algébrique (Louvain, 1956), Georges Thone, Liège

3. Bhanot, G., Fucito, F., Solomon, S.: $S U(3)$ topological charge on the lattice. Phys. Lett. B194, 114-118 (1987)

4. Brown, E.: Twisted tensor products I. Ann. Math. 69, 223-246 (1959)

5. Buchberger, B.: Gröbner bases: An algorithmic model in polynomial ideal theory. In: Multidimensional Systems Theory. Bose, N. K. (ed), Dordrecht: Reidel 1985

6. Chern, S.: Complex Manifolds without Potential Theory. 2nd Ed. Berlin, Heidelberg, New York: Springer 1979

7. Fabricius, K., Rossi, G. C.: Monte Carlo measurement of the topological susceptibility in SU(3) lattice gauge theory. Phys. Lett. 127B, 229-232 (1983)

8. Göckeler, M., Kronfeld, A. S., Laursen, M. L., Shierholz, G., Wiese, U.-J.: Topology of $S U(3)$ lattice gauge theory. Nucl. Phys. B292, 349-362 (1987)

9. Griffiths, P., Harris, J.: Principles of Algebraic Geometry. New York: Wiley 1978

10. Hoek, J.: Cooling of $S U(3)$ lattice gauge field configurations and the $\eta^{\prime}$ mass. Phys. Lett 166B, 199-202 (1986)

11. Kobayashi, S., Nomizu, K.: Foundations of Differential Geometry I. New York: Interscience 1963 
12. Kronfeld, A. S.: Topological aspects of lattice gauge theory. In: Proceedings, Lattice 87 Conference, Seillac, Nucl. Phys. B [Proc. Suppl.] 4, 239-351 (1988)

13. Laursen, M.: $S U(3)$ lattice topology: A new, fast geometric charge algorithm. In: Proceedings, Fermilab Symposium on Lattice Field Theory. Kronfeld, A. S., Mackenzie, P. D. (eds.). Nucl. Phys. B [Proc. Suppl.], 9, 411-415 (1989)

14. Lasher, G.: The winding number of 3-complexes in $S U(3)$. In: Proceedings, Fermilab Symposium on Lattice Field Theory. Kronfeld, A. S., Mackenzie, P. D. (eds.). Nucl. Phys. B [Proc. Suppl.] 9, 416-421 (1989)

15. Lasher, G.: Winding numbers of $S U(3)$ lattice gauge fields (to appear in Ann. Phys.)

16. Li, T., Sauer, T., Yorke, J.: Numerically determining solutions of systems of polynomial equations. Bull. Am. Math. Soc. (NS) 18, 173-177 (1988)

17. Lüscher, M.: Topology of lattice gauge fields. Commun. Math. Phys. 85, 39-48 (1982)

18. Milnor, J.: Construction of universal bundles I. Ann. Math. 63, 272-284 (1956)

19. Milnor, J.: Construction of universal bundles II. Ann. Math. 63, 430-436 (1956)

20. Milnor, J.: Singular Points of Complex Hypersurfaces, Annals of Math. Studies No. 61. Princeton, NJ: Princeton University Press 1968

21. Milnor, J., Stasheff, J.: Characteristic Classes. Annals of Math. Studies vol. 76. Princeton, NJ: Princeton University Press 1974

22. Parisi, G., Rapuano, F.: On the $S U(3)$ topological charge in lattice gauge theories. Phys. Lett. 152B, 218 (1985)

23. Phillips, A.: Characteristic numbers of $U_{1}$-valued lattice gauge fields. Ann. Phys. 161, 399-422 (1985)

24. Phillips, A., Stone, D.: Lattice gauge fields, principal bundles, and the calculation of topological charge. Commun. Math. Phys. 103, 599-636 (1986)

25. Robbiano, L. (ed),: Computational Aspects of Commutative Algebra. New York: Academic Press 1989

26. Steenrod, N.: Topology of Fibre Bundles. Princeton: Princeton University Press 1951

Communicated by K. Gawedzki

Received April 12, 1989; in revised form December 2, 1989 